\title{
An expedient synthesis of new 2-(furoxan-3-yl)thiazolidin-4-one derivatives
}

\author{
Singam Naveen Kumar, ${ }^{a}$ Chebolu Naga Sesha Sai Pavan Kumar, ${ }^{* a, c}$ \\ Sri Ranga Vanarasi Anudeep, ${ }^{a}$ Kirti Kumari Sharma, ${ }^{\text {a,b }}$ Vaidya Jayathirtha Rao, ${ }^{\text {a,b }}$ \\ and Nanubolu Jagadeesh Babu ${ }^{d}$ \\ ${ }^{a}$ Crop Protection Chemicals Division, Indian Institute of Chemical Technology, \\ Uppal Road Tarnaka, Hyderabad 500 007, Telangana, India \\ ${ }^{b}$ AcSIR-IICT, CSIR-Indian Institute of Chemical Technology, Uppal Road Tarnaka, \\ Hyderabad 500 007, Telangana, India \\ ${ }^{c}$ Division of Chemistry, Department of Sciences and Humanities, \\ Vignan's Foundation for Science, Technology \& Research, Vignan University, \\ Vadlamudi, Guntur 522 213, Andhra Pradesh, India \\ ${ }^{d}$ Laboratory of X-ray Crystallography, Indian Institute of Chemical Technology, \\ Hyderabad 500 007, India. \\ E-mail:pavaniict@gmail.com
}

DOI: http://dx.doi.org/10.3998/ark.5550190.p009.669

\begin{abstract}
A series of new biologically interesting furoxan-3-thiazolidinones have been synthesized via onepot three-component reaction of furoxan aldehydes, anilines and mercaptoacetic acid. The multicomponent reaction involves condensation of furoxan aldehyde with aniline to give imine; the formed imine undergoes nucleophilic addition with mercaptoacetic acid, followed by cyclisation with loss of $\mathrm{H}_{2} \mathrm{O}$ to obtain the desired products. All the synthesized compounds were well characterized using spectral techniques and confirmed by an X-ray crystal structure for one compound.
\end{abstract}

Keywords: Furoxan-3-carbaldehydes, One-pot reaction, thiazolidin-4-ones, crystal structure

\section{Introduction}

To fight against disease, society depends on the development of new biologically active compounds. One of the new approaches towards this goal is the development of hybrid heterocyclic compounds. A large number of chemical libraries can be accessed by the combination 
of different heterocycles and they may be considered to be valuable as they incorporate classes of compound with proven utility in medicinal chemistry.

Furoxans (1,2,5-oxadiazole 2-oxides) are a class of five membered heterocyclic compounds having oxygen and nitrogen as heteroatoms. They constitute an important class of heterocyclic compounds possessing biological activities such as antihelmintic, antitumor, antimicrobial, mutagenic, immunosuppressive, anticancer, and anti-aggregating properties. ${ }^{1-3}$ Some furoxan derivatives shown potential cardiovascular properties. ${ }^{4}$ Furthermore, furoxans are well known as NO-donors, and recently furoxan compounds have shown activity against schistosomiasis. ${ }^{5}$ Furoxans are also used in combination with drugs as NO donor-drug hybrids. $\alpha 1$-Antagonists, $\beta 1$ antagonists, $\mathrm{Ca}^{2+}$-channel blockers, $\mathrm{K}^{+}$-channel activators, NSAIDs, and $\mathrm{H} 3$ - and $\mathrm{H} 2$-antagonists are a few examples having NO donor-drug hybrids. ${ }^{6} \mathrm{NO}$ donor-1,4-dihydropyridine also proved to be $\mathrm{Ca}^{2+}$-channel activators. ${ }^{7}$ Furthermore, REC15/2739, a uroselective $\alpha 1$-antagonist, ${ }^{8}$ and Rabeprazole, a potent inhibitor of $\mathrm{H}^{+} / \mathrm{K}^{+}$-ATPase enzyme, ${ }^{9}$ are also NO donor-drugs.

Thiazolidinones and related structures are present in natural products; they have a wide range of biological activities and comprise an important motif in pharmaceutical compounds. ${ }^{10-13}$ Thiazolidinones and their derivatives have been reported to possess anticonvulsant, ${ }^{14-16}$ antifungal, ${ }^{17-19}$ antitubercular, ${ }^{20,21}$ antitumour, ${ }^{22}$ antiparasitic, ${ }^{23}$ herbicidal, ${ }^{24}$ anti-inflammatory, ${ }^{25}$ analgesic, ${ }^{26}$ anticancer, ${ }^{27,28}$ antibacterial, ${ }^{29-31}$ and antipsychotic ${ }^{32}$ properties. They have also been reported to inhibit the bacterial enzyme Mur-B, a precursor in the biosynthesis of peptidoglycon, which is a non-nucleoside inhibitor of HIV-RT. ${ }^{33,34}$ Motivated by these findings, and in continuation of our ongoing efforts towards with the discovery of nitrogenated heterocycles with potential chemotherapeutic activities, ${ }^{35,36}$ we planned to synthesize a new series of hybrid furoxan3-thiazolidinone derivatives of potential biological activity.

\section{Results and Discussion}

The present work was designed to synthesize new furoxan-3-thiazolidinones from substituted furoxan aldehydes (4a-c). The synthetic scheme was depicted in Scheme 1. The vital intermediates, furoxan aldehydes 4a-c, were synthesized following a known route from substituted benzaldehydes. Horner Wadsworth Emmons reaction of benzaldehydes in the presence of $\mathrm{NaH}$ as a base yielded substituted ethyl cinnamates 1a-c. The ethyl cinnamates were subjected to DIBAL$\mathrm{H}$ reduction to yield cinnamyl alcohols $2 \mathbf{2 a - c}$. Further, the cinnamyl alcohols were treated with aq. $\mathrm{NaNO}_{2}$ in the presence of glacial acetic acid to obtain furoxan methanol derivatives 3a-c. ${ }^{37}$ Oxidation of the alcohol functionality of the furoxanmethanols using $\mathrm{MnO}_{2}$ yielded furoxan aldehydes 4a-c in excellent yields. ${ }^{37}$ Finally, a one-pot three component reaction of furoxan aldehydes 4a-c with substituted anilines 6a-n and mercaptoacetic acid $\mathbf{5}$ was achieved by simple heating in toluene at $50{ }^{\circ} \mathrm{C}$ to obtain the required furoxan-3-thiazolidinones $7 \mathbf{a}-\mathbf{n}, \mathbf{8 a}-\mathbf{n}, 9 \mathbf{a}-\mathbf{n}$ in good yields. 
<smiles>[R]c1ccc(C=O)cc1</smiles><smiles>[R]OCOCF</smiles>

1a: $\mathrm{R}=\mathrm{H}$

1b: $\mathrm{R}=\mathrm{OMe}$

1c: $R=F$

2a: $\mathrm{R}=\mathrm{H}$

2b: $\mathrm{R}=\mathrm{OMe}$

2c: $R=F$<smiles>[R]c1ccc(-c2no[n+]([O-])c2CO)cc1</smiles><smiles>[R]c1ccc(-c2no[n+]([O-])c2C=O)cc1</smiles>

3a: $\mathrm{R}=\mathrm{H}$

3b: $\mathrm{R}=\mathrm{OMe}$

3c: $R=F$

4a: $\mathrm{R}=\mathrm{H}$

4b: $\mathrm{R}=\mathrm{OMe}$

4c: $R=F$<smiles>[R]c1ccc(-c2no[n+]([O-])c2C=O)cc1</smiles>

4a-c<smiles>O=C([O-])CS</smiles>

5<smiles>[R]c1ccc(-c2no[n+]([O-])c2C2SCC(=O)N2c2ccc([R])cc2)cc1</smiles>

6a-n

7a-n, 8a-n, 9a-n

Reagents and conditions: a $(\mathrm{EtO}){ }_{2} \mathrm{P}(\mathrm{O}) \mathrm{CH}_{2} \mathrm{COOEt}, \mathrm{NaH}, \mathrm{DCM}, 0{ }^{\circ} \mathrm{C}-\mathrm{rt}, 1 \mathrm{~h}, 85-90 \%$. $b$ DIBAL-H, DCM, $0{ }^{\circ} \mathrm{C}, 4$ h. $c$ Acetic acid, aq. $\mathrm{NaNO}_{2}$, rt, 4-6 h, 30-75\%. $d \mathrm{MnO}_{2}, \mathrm{CH}_{2} \mathrm{Cl}_{2}, 2-4 \mathrm{~h}, 85-$ $95 \%$ e Toluene, $50^{\circ} \mathrm{C}, 4 \mathrm{~h}, 70-84 \%$.

Scheme 1. The preparation of furoxanyl thiazolidinones 7-9.

Multi-component reaction (MCR) involves three main steps. In step 1, the condensation of furoxan aldehyde with aniline forms imine I; the imine undergoes nucleophilic addition with mercaptoacetic acid to give intermediate II (step 2), followed by cyclisation (by loss of $\mathrm{H}_{2} \mathrm{O}$ ) to give the final product (step 3) as shown in Scheme 2. All the synthesized compounds are listed in Table 1.

The structural connectivity of the furoxan thiazolidinones is confirmed by a single-crystal Xray diffraction analysis of compound $\mathbf{7 g}$, as shown in Figure 1. 
<smiles>O=Cc1c(-c2ccccc2)no[n+]1[O-]</smiles>

Furoxan aldehyde<smiles>Nc1ccccc1</smiles>

Aniline

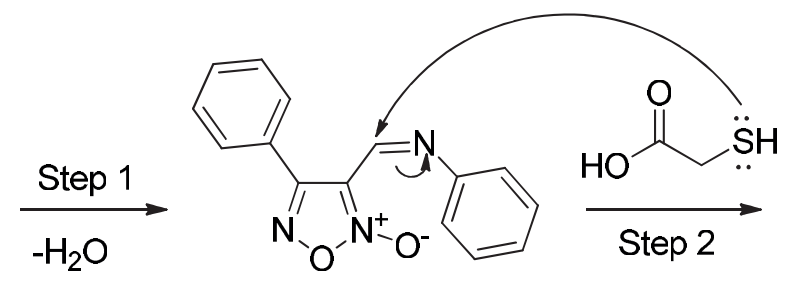

I

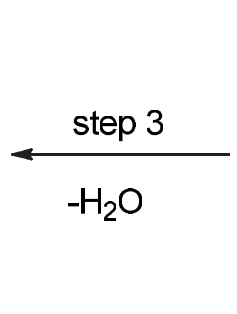

Product

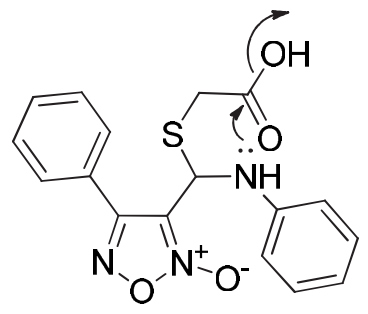

II

Scheme 2. Suggested pathway to thiazolidinone ring formation.

\section{X-ray Crystallographic analysis ${ }^{38,39}$}

Data for 7g, a colorless crystal compound, molecular weight: $\mathrm{C}_{18} \mathrm{H}_{15} \mathrm{~N}_{3} \mathrm{O}_{4} \mathrm{~S}, M=369.394$, colorless block, $0.38 \times 0.34 \times 0.26 \mathrm{~mm}^{3}$, monoclinic, space group $P 2_{1} / n$ (No. 14), $a=8.9714(7), b=$ $16.3622(13), c=11.8077(9) \AA, \beta=98.4520(10)^{\circ}, V=1714.4(2) \AA^{3}, Z=4, D_{\mathrm{c}}=1.431 \mathrm{~g} / \mathrm{cm}^{3}, F_{000}$ $=768, \mathrm{CCD}$ area detector, MoK $\alpha$ radiation, $\lambda=0.71073 \AA, T=293(2) \mathrm{K}, 2 \theta_{\max }=50.0^{\circ}, 16244$ reflections collected, 3020 unique $\left(\mathrm{R}_{\text {int }}=0.0192\right)$, Final $G o o F=1.030, R 1=0.0312, w R 2=0.0845$, $R$ indices based on 2767 reflections with $\mathrm{I}>2 \sigma(\mathrm{I})$ (refinement on $F^{2}$ ), 237 parameters, $\mu=0.219$ $\mathrm{mm}^{-1}$. 


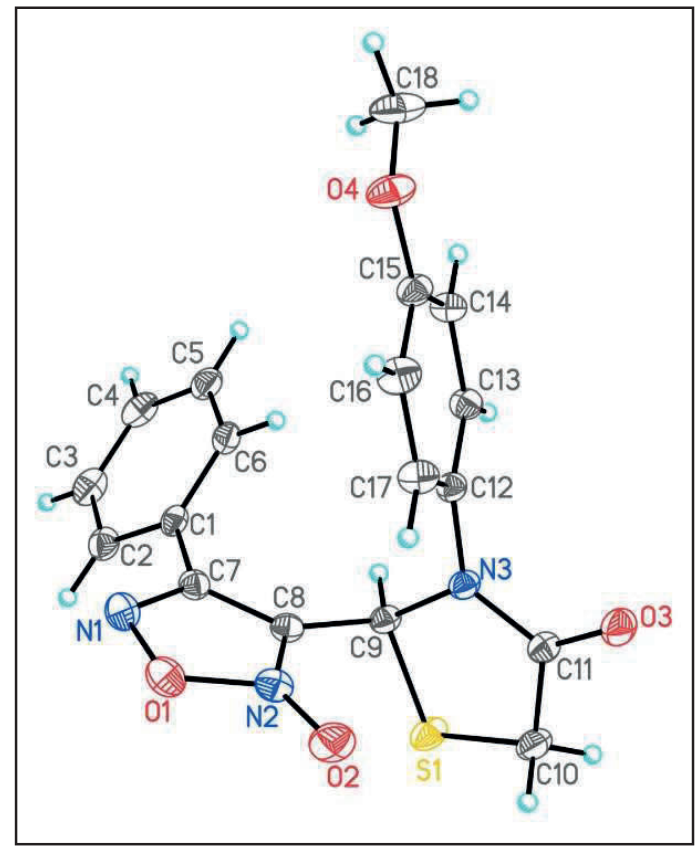

Figure 1. ORTEP diagram of the compound $7 \mathrm{~g}$.

Table 1. One-pot synthesis of 2-(furoxan-3-yl)thiazolidin-4-ones<smiles>[R]c1ccc(-c2no[n+]([O-])c2C=O)cc1</smiles>

4a: $\mathrm{R}=\mathrm{H}$ 4b: $\mathrm{R}=\mathrm{OMe}$ 4c: $R=F$<smiles>O=C(O)CS</smiles>

5

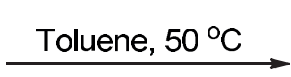<smiles>[R]c1ccc(-c2no[n+]([O-])c2C2SCC(=O)N2c2ccc([R])cc2)cc1</smiles>

7a-n, 8a-n, 9a-n

\begin{tabular}{cccccc}
\hline Entry & $\begin{array}{c}\text { Furoxan } \\
\text { aldehyde }\end{array}$ & Substituted Aniline & Product & $\begin{array}{c}\text { Isolated } \\
\text { yield }(\%)\end{array}$ & $\begin{array}{c}\text { Melting point } \\
\left({ }^{\circ} \mathrm{C}\right)\end{array}$ \\
\hline 1 & $\mathbf{4 a}$ & $\mathrm{R}^{\prime}=\mathrm{H}(\mathbf{6 a})$ & $\mathrm{R}, \mathrm{R}^{\prime}=\mathrm{H}(\mathbf{7 a})$ & 82 & $82-84$ \\
2 & $\mathbf{4 a}$ & $\mathrm{R}^{\prime}=4-n-\mathrm{Bu}(\mathbf{6 b})$ & $\mathrm{R}=\mathrm{H} ; \mathrm{R}^{\prime}=4-n-\mathrm{Bu}(\mathbf{7 b})$ & 77 & $100-102$ \\
3 & $\mathbf{4 a}$ & $\mathrm{R}^{\prime}=4-i-\mathrm{Pr}(\mathbf{6 c})$ & $\mathrm{R}=\mathrm{H} ; \mathrm{R}^{\prime}=4-i-\mathrm{Pr}(\mathbf{7 c})$ & 75 & $160-164$ \\
4 & $\mathbf{4 a}$ & $\mathrm{R}^{\prime}=4-t-\mathrm{Bu}(\mathbf{6 d})$ & $\mathrm{R}=\mathrm{H} ; \mathrm{R}^{\prime}=4-t-\mathrm{Bu}(\mathbf{7 d})$ & 81 & $150-152$ \\
5 & $\mathbf{4 a}$ & $\mathrm{R}^{\prime}=3,5-\operatorname{diMe}(\mathbf{6 e})$ & $\mathrm{R}=\mathrm{H} ; \mathrm{R}^{\prime}=3,5-\operatorname{diMe}(\mathbf{7 e})$ & 78 & $160-161$ \\
6 & $\mathbf{4 a}$ & $\mathrm{R}^{\prime}=3,4-\operatorname{diMe}(\mathbf{6 f})$ & $\mathrm{R}=\mathrm{H} ; \mathrm{R}^{\prime}=3,4-\operatorname{diMe}(\mathbf{7 f})$ & 78 & $145-147$ \\
7 & $\mathbf{4 a}$ & $\mathrm{R}^{\prime}=4-\mathrm{OMe}(\mathbf{6 g})$ & $\mathrm{R}=\mathrm{H} ; \mathrm{R}^{\prime}=4-\mathrm{OMe}(\mathbf{7 g})$ & 78 & $129-131$ \\
8 & $\mathbf{4 a}$ & $\mathrm{R}^{\prime}=3,4-\operatorname{diOMe}(\mathbf{6 h})$ & $\mathrm{R}=\mathrm{H} ; \mathrm{R}^{\prime}=3,4-\operatorname{diOMe}(\mathbf{7 h})$ & 77 & $120-124$ \\
9 & $\mathbf{4 a}$ & $\mathrm{R}^{\prime}=3,4-\mathrm{OCH} \mathrm{H}_{2} \mathrm{O}(\mathbf{6 i})$ & $\mathrm{R}=\mathrm{H} ; \mathrm{R}^{\prime}=3,4-\mathrm{OCH} \mathrm{H}_{2} \mathrm{O}(\mathbf{7 i})$ & 77 & $121-123$ \\
10 & $\mathbf{4 a}$ & $\mathrm{R}^{\prime}=3-\mathrm{F}(\mathbf{6 j})$ & $\mathrm{R}=\mathrm{H} ; \mathrm{R}^{\prime}=3-\mathrm{F} \mathbf{( 7 \mathbf { j } )}$ & 77 & $99-101$ \\
\hline
\end{tabular}


Table 1 (continued)

\begin{tabular}{|c|c|c|c|c|c|}
\hline Entry & $\begin{array}{l}\text { Furoxan } \\
\text { aldehyde }\end{array}$ & Substituted Aniline & Product & $\begin{array}{c}\text { Isolated } \\
\text { yield (\%) }\end{array}$ & $\begin{array}{l}\text { Melting point } \\
\left({ }^{\circ} \mathrm{C}\right)\end{array}$ \\
\hline 11 & $4 a$ & $\mathrm{R}^{\prime}=4-\mathrm{F}(6 \mathbf{k})$ & $\mathrm{R}=\mathrm{H} ; \mathrm{R}^{\prime}=4-\mathrm{F}(\mathbf{7 k})$ & 77 & $118-120$ \\
\hline 12 & $4 a$ & $\mathrm{R}^{\prime}=3-\mathrm{Cl}(\mathbf{6 1})$ & $\mathrm{R}=\mathrm{H} ; \mathrm{R}^{\prime}=3-\mathrm{Cl}(\mathbf{7 l})$ & 80 & $108-110$ \\
\hline 13 & $4 a$ & $\mathrm{R}^{\prime}=4-\mathrm{Cl}(6 \mathrm{~m})$ & $\mathrm{R}=\mathrm{H} ; \mathrm{R}^{\prime}=4-\mathrm{Cl}(\mathbf{7 m})$ & 81 & $158-159$ \\
\hline 14 & $4 a$ & $\mathrm{R}^{\prime}=4-\mathrm{Br}(\mathbf{6 n})$ & $\mathrm{R}=\mathrm{H} ; \mathrm{R}^{\prime}=4-\mathrm{Br}(\mathbf{7 n})$ & 70 & $198-200$ \\
\hline 15 & $4 b$ & $\mathrm{R}^{\prime}=\mathrm{H}(\mathbf{6} \mathbf{a})$ & $\mathrm{R}=\mathrm{OMe} \mathrm{R}^{\prime}=\mathrm{H}(\mathbf{8} \mathbf{a})$ & 80 & $150-152$ \\
\hline 16 & $4 b$ & $\mathrm{R}^{\prime}=4-n-\mathrm{Bu}(\mathbf{6 b})$ & $\mathrm{R}=\mathrm{OMe} ; \mathrm{R}^{\prime}=4-n-\mathrm{Bu}(\mathbf{8 b})$ & 81 & $99-100$ \\
\hline 17 & $4 b$ & $\mathrm{R}^{\prime}=4-i-\operatorname{Pr}(\mathbf{6 c})$ & $\mathrm{R}=\mathrm{OMe} ; \mathrm{R}^{\prime}=4-i-\operatorname{Pr}(\mathbf{8 c})$ & 77 & $125-127$ \\
\hline 18 & $4 b$ & $\mathrm{R}^{\prime}=4-t-\mathrm{Bu}(\mathbf{6 d})$ & $\mathrm{R}=\mathrm{OMe} ; \mathrm{R}^{\prime}=4-t-\mathrm{Bu}(\mathbf{8 d})$ & 81 & $126-128$ \\
\hline 19 & $4 b$ & $\mathrm{R}^{\prime}=3,5-\operatorname{diMe}(\mathbf{6 e})$ & $\mathrm{R}=\mathrm{OMe} ; \mathrm{R}^{\prime}=3,5-\mathrm{diMe}(\mathbf{8 e})$ & 84 & $128-130$ \\
\hline 20 & $4 b$ & $\mathrm{R}^{\prime}=3,4-\operatorname{diMe}(\mathbf{6 f})$ & $\mathrm{R}=\mathrm{OMe} ; \mathrm{R}^{\prime}=3,4-\operatorname{diMe}(\mathbf{8 f})$ & 78 & $120-122$ \\
\hline 21 & $4 b$ & $\mathrm{R}^{\prime}=4-\mathrm{OMe}(\mathbf{6 g})$ & $\mathrm{R}=\mathrm{OMe} ; \mathrm{R}^{\prime}=4-\mathrm{OMe}(\mathbf{8 g})$ & 78 & $112-114$ \\
\hline 22 & $4 b$ & $\mathrm{R}^{\prime}=3,4-\mathrm{diOMe}(\mathbf{6 h})$ & $\mathrm{R}=\mathrm{OMe} ; \mathrm{R}^{\prime}=3,4-\mathrm{diOMe}(\mathbf{8 h})$ & 78 & $128-130$ \\
\hline 23 & $4 b$ & $\mathrm{R}^{\prime}=3,4-\mathrm{OCH}_{2} \mathrm{O}(\mathbf{6 i})$ & $\mathrm{R}=\mathrm{OMe} ; \mathrm{R}^{\prime}=3,4-\mathrm{OCH}_{2} \mathrm{O}(\mathbf{8 i})$ & 76 & $165-168$ \\
\hline 24 & $4 b$ & $\mathrm{R}^{\prime}=3-\mathrm{F}(\mathbf{6} \mathbf{j})$ & $\mathrm{R}=\mathrm{OMe} ; \mathrm{R}^{\prime}=3-\mathrm{F}(\mathbf{8} \mathbf{j})$ & 74 & $120-122$ \\
\hline 25 & $4 b$ & $\mathrm{R}^{\prime}=4-\mathrm{F}(\mathbf{6 k})$ & $\mathrm{R}=\mathrm{OMe} ; \mathrm{R}^{\prime}=4-\mathrm{F}(\mathbf{8} \mathbf{k})$ & 74 & $110-112$ \\
\hline 26 & $4 b$ & $\mathrm{R}^{\prime}=3-\mathrm{Cl}(\mathbf{6 l})$ & $\mathrm{R}=\mathrm{OMe} ; \mathrm{R}^{\prime}=3-\mathrm{Cl}(\mathbf{8 1})$ & 76 & $125-128$ \\
\hline 27 & $4 b$ & $\mathrm{R}^{\prime}=4-\mathrm{Cl}(6 \mathrm{~m})$ & $\mathrm{R}=\mathrm{OMe} ; \mathrm{R}^{\prime}=4-\mathrm{Cl}(\mathbf{8 m})$ & 77 & $123-125$ \\
\hline 28 & $4 b$ & $\mathrm{R}^{\prime}=4-\mathrm{Br}(\mathbf{6 n})$ & $\mathrm{R}=\mathrm{OMe} ; \mathrm{R}^{\prime}=4-\mathrm{Br}(\mathbf{8 n})$ & 70 & $122-124$ \\
\hline 29 & $4 c$ & $\mathrm{R}^{\prime}=\mathrm{H}(\mathbf{6} \mathbf{a})$ & $\mathrm{R}=\mathrm{F} ; \mathrm{R}^{\prime}=\mathrm{H}(\mathbf{9 a})$ & 82 & $108-110$ \\
\hline 30 & $4 c$ & $\mathrm{R}^{\prime}=4-n-\mathrm{Bu}(\mathbf{6 b})$ & $\mathrm{R}=\mathrm{F} ; \mathrm{R}^{\prime}=4-n-\mathrm{Bu}(\mathbf{9 b})$ & 78 & $90-93$ \\
\hline 31 & $4 c$ & $\mathrm{R}^{\prime}=4-i-\operatorname{Pr}(\mathbf{6 c})$ & $\mathrm{R}=\mathrm{F} ; \mathrm{R}^{\prime}=4-i-\operatorname{Pr}(\mathbf{9 c})$ & 78 & $160-161$ \\
\hline 32 & $4 c$ & $\mathrm{R}^{\prime}=4-t-\mathrm{Bu}(\mathbf{6 d})$ & $\mathrm{R}=\mathrm{F} ; \mathrm{R}^{\prime}=4-t-\mathrm{Bu}(\mathbf{9 d})$ & 76 & $175-178$ \\
\hline 33 & $4 c$ & $\mathrm{R}^{\prime}=3,5-\operatorname{diMe}(\mathbf{6 e})$ & $\mathrm{R}=\mathrm{F} ; \mathrm{R}^{\prime}=3,5-\operatorname{diMe}(\mathbf{9 e})$ & 76 & $178-180$ \\
\hline 34 & $4 c$ & $\mathrm{R}^{\prime}=3,4-\operatorname{diMe}(\mathbf{6 f})$ & $\mathrm{R}=\mathrm{F} ; \mathrm{R}^{\prime}=3,4-\operatorname{diMe}(\mathbf{9 f})$ & 79 & $145-147$ \\
\hline 35 & $4 c$ & $\mathrm{R}^{\prime}=4-\mathrm{OMe}(\mathbf{6 g})$ & $\mathrm{R}=\mathrm{F} ; \mathrm{R}^{\prime}=4-\mathrm{OMe}(\mathbf{9 g})$ & 77 & $142-144$ \\
\hline 36 & $4 c$ & $\mathrm{R}^{\prime}=3,4-\operatorname{diOMe}(\mathbf{6 h})$ & $\mathrm{R}=\mathrm{F} ; \mathrm{R}^{\prime}=3,4-\mathrm{diOMe}(\mathbf{9 h})$ & 77 & $160-163$ \\
\hline 37 & $4 c$ & $\mathrm{R}^{\prime}=3,4-\mathrm{OCH}_{2} \mathrm{O}(\mathbf{6 i})$ & $\mathrm{R}=\mathrm{F} ; \mathrm{R}^{\prime}=3,4-\mathrm{OCH}_{2} \mathrm{O}(\mathbf{9 i})$ & 74 & $180-182$ \\
\hline 38 & $4 c$ & $\mathrm{R}^{\prime}=3-\mathrm{F}(\mathbf{6} \mathbf{j})$ & $\mathrm{R}=\mathrm{F} ; \mathrm{R}^{\prime}=3-\mathrm{F}(\mathbf{9 j})$ & 73 & $128-131$ \\
\hline 39 & $4 \mathrm{c}$ & $\mathrm{R}^{\prime}=4-\mathrm{F}(\mathbf{6 k})$ & $\mathrm{R}=\mathrm{F} ; \mathrm{R}^{\prime}=4-\mathrm{F}(\mathbf{9 k})$ & 74 & $148-150$ \\
\hline 40 & $4 c$ & $\mathrm{R}^{\prime}=3-\mathrm{Cl}(\mathbf{6 l})$ & $\mathrm{R}=\mathrm{F} ; \mathrm{R}^{\prime}=3-\mathrm{Cl}(9 \mathrm{l})$ & 74 & $105-107$ \\
\hline 41 & $4 \mathrm{c}$ & $\mathrm{R}^{\prime}=4-\mathrm{Cl}(6 \mathrm{~m})$ & $\mathrm{R}=\mathrm{F} ; \mathrm{R}^{\prime}=4-\mathrm{Cl}(\mathbf{9 m})$ & 82 & $109-110$ \\
\hline 42 & $4 c$ & $\mathrm{R}^{\prime}=4-\mathrm{Br}(\mathbf{6 n})$ & $R=F ; R^{\prime}=4-B r(9 n)$ & 70 & $147-150$ \\
\hline
\end{tabular}




\section{Conclusions}

Syntheses of a number of new furoxan-3-thiazolidinones were successfully accomplished and are well characterized by spectral data. The anticancer activity of these compounds is being studied and will be reported in due course.

\section{Experimental Section}

General. All reactions involving air-sensitive reagents were performed under nitrogen atmosphere. Solvents were freshly dried and purified by conventional methods prior to use. The progress of all the reactions was monitored by TLC, using TLC aluminium sheets precoated with silica gel $60 \mathrm{~F}_{254}$ to a thickness of $0.25 \mathrm{~mm}$ (Merck). Flash column chromatography was done using silica gel (Merck, 60-120 mesh). Melting points were determined on a MEL-TEMP II melting point apparatus. IR spectra were recorded on a Perkin-Elmer FT-IR spectrophotometer and expressed with $\left(v_{\max }, \mathrm{cm}^{-1}\right) .{ }^{1} \mathrm{H}$ and ${ }^{13} \mathrm{C}$ NMR spectra were recorded on a Varian Gemini 200 $\mathrm{MHz}$, Bruker Avance $300 \mathrm{MHz}$ spectrometer; TMS was used as an internal standard in $\mathrm{CDCl}_{3} / \mathrm{DMSO}-\mathrm{d}_{6}$. Mass spectra were recorded on VG Micromass $7070 \mathrm{H}$ (EI), QSTAR XL High resolution mass spectrometer (HRMS), Thermofinnigan ESI ion trap Mass Spectrometer and a GC-MS system on an Agilent 6890 series.

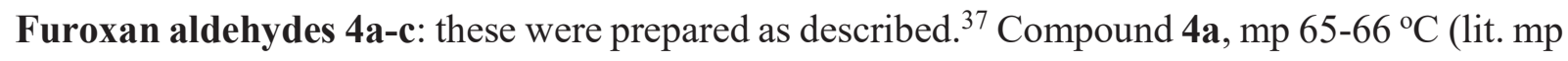
$64-65^{\circ} \mathrm{C}$ ); $\mathbf{4 b}$, mp $92-94{ }^{\circ} \mathrm{C}$; $\mathbf{4 c}, 74-76^{\circ} \mathrm{C}$ (no lit. ${ }^{37} \mathrm{mps}$ were reported for $\mathbf{4 b , c}$ ).

General procedure for the preparation of furoxan-3-thiazolidinones. To the solution of furoxan aldehyde $(2.5 \mathrm{mmol})$ in $10 \mathrm{~mL}$ toluene, was added aniline $(2.75 \mathrm{mmol})$ in $10 \mathrm{~mL}$ of toluene at $0{ }^{\circ} \mathrm{C}$. After formation of imine which is monitored by TLC, thioglycolic acid (10 mmol) was added to the reaction mixture at the same temperature. The reaction was stirred and heated at 50 ${ }^{\circ} \mathrm{C}$ for $4 \mathrm{~h}$. After completion of the reaction, the reaction mixture was diluted with ethyl acetate and washed with saturated solution of $\mathrm{NaHCO}_{3}(3 \times 20 \mathrm{~mL})$. The combined organic layer was dried $\left(\mathrm{Na}_{2} \mathrm{SO}_{4}\right)$, concentrated under reduced pressure. The crude products were purified using silica-gel column chromatography (eluent-3:2 hexane-ethyl acetate), to afford pure products.

3-(4-Oxo-3-phenylthiazolidin-2-yl)-4-phenyl-1,2,5-oxadiazole 2-oxide (7a). White solid (82\%), m.p. $82-84^{\circ} \mathrm{C}$, IR (film): 2924, 2853, 1703, 1596, 1492, 1455, 1377, 1218, 834, $770 \mathrm{~cm}^{-1} .{ }^{1} \mathrm{H} \mathrm{NMR}$ $\left(300 \mathrm{MHz}, \mathrm{CDCl}_{3}\right) \delta$ 7.60-7.45 (m, 3H), 7.40-7.24 (m, 5H), 7.16-7.10 (m, 2H), $6.17(\mathrm{~s}, 1 \mathrm{H}), 4.04$ (brd, $1 \mathrm{H}, J 15.2 \mathrm{~Hz}), 3.77$ (d, $1 \mathrm{H}, J 15.2 \mathrm{~Hz}) .{ }^{13} \mathrm{C} \mathrm{NMR}\left(75 \mathrm{MHz}, \mathrm{CDCl}_{3}\right) \delta 170.1,155.7,136.0$, 131.3, 129.7, 129.2, 128.4, 127.9, 125.3, 125.1, 114.4, 54.6, 33.7. ESI-MS: $m / z 362(\mathrm{M}+\mathrm{Na})^{+}$. HRMS (ESI): $m / z[\mathrm{M}+\mathrm{Na}]^{+}$Calcd for $\mathrm{C}_{17} \mathrm{H}_{13} \mathrm{~N}_{3} \mathrm{O}_{3} \mathrm{SNa}$ : 362.0570 ; Found: 362.0588 .

3-[3-(4-Butylphenyl)-4-oxothiazolidin-2-yl]-4-phenyl-1,2,5-oxadiazole 2-oxide (7b). White solid (77\%), m.p. 100-102 ${ }^{\circ} \mathrm{C}$, IR (film): 3036, 2927, 2856, 1693, 1594, 1453, 1341, 782, $759 \mathrm{~cm}^{-}$ 
1. ${ }^{1} \mathrm{H}$ NMR $\left(300 \mathrm{MHz}, \mathrm{CDCl}_{3}\right) \delta 7.55$ (tt, $1 \mathrm{H}, J$ 7.4, $\left.1.8 \mathrm{~Hz}\right), 7.50-7.45$ (m, 2H), 7.24 (brd, 2H, $J$ $7.4 \mathrm{~Hz}), 7.16(\mathrm{dd}, 2 \mathrm{H}, J 8.3 \mathrm{~Hz}), 7.02(\mathrm{dd}, 2 \mathrm{H}, J 8.3 \mathrm{~Hz}), 6.11(\mathrm{~s}, 1 \mathrm{H}), 4.02$ (brd, $1 \mathrm{H}, J 15.1 \mathrm{~Hz})$, $3.74(\mathrm{~d}, 1 \mathrm{H}, J 15.1 \mathrm{~Hz}), 2.57$ (t, 2H, J 7.7 Hz), 1.62-1.52 (m, 2H), 1.36-1.26 (m, 2H), 0.90 (t, 3H, $J 7.3 \mathrm{~Hz}) .{ }^{13} \mathrm{C}$ NMR $\left(75 \mathrm{MHz}, \mathrm{CDCl}_{3}\right) \delta 170.2,155.8,143.6,133.5,131.3,129.7,129.2,128.0$, 125.3, 125.2, 114.4, 54.8, 35.1, 33.7, 33.2, 22.1, 13.8. ESI-MS: $m / z 396(\mathrm{M}+\mathrm{H})^{+}$. HRMS (ESI): $m / z[\mathrm{M}+\mathrm{H}]^{+}$Calcd for $\mathrm{C}_{21} \mathrm{H}_{22} \mathrm{~N}_{3} \mathrm{O}_{3} \mathrm{~S}: 396.1376$; Found: 396.1380 .

3-[3-(4-Isopropylphenyl)-4-oxothiazolidin-2-yl]-4-phenyl-1,2,5-oxadiazole 2-oxide (7c). White solid (75\%), m.p. 160-164 ${ }^{\circ} \mathrm{C}$, IR (film): 3385, 2964, 2368, 1701, 1591, 1224, $700 \mathrm{~cm}^{-1} .{ }^{1} \mathrm{H}$ NMR (300 MHz, $\left.\mathrm{CDCl}_{3}\right) \delta$ 7.63-7.44 (m, 3H), 7.29-7.17 (m, 4H), 7.03 (d, 2H, J 8.3 Hz), 6.10 (s, 1H), 4.02 (brd, 1H, $J 15.1 \mathrm{~Hz}), 3.74$ (d, 1H, $J 15.1 \mathrm{~Hz}), 2.88$ (sept, 1H, $J 6.7 \mathrm{~Hz}), 1.21$ (d, 6H, $J$ $6.7 \mathrm{~Hz}) .{ }^{13} \mathrm{C}$ NMR $\left(75 \mathrm{MHz}, \mathrm{CDCl}_{3}\right) \delta 170.2,155.8,149.4,133.5,131.2,129.2,128.0,127.8$, 125.4, 125.2, 114.4, 54.7, 33.7 (2C), 23.7. ESI-MS: $m / z 382(\mathrm{M}+\mathrm{H})^{+}$. HRMS (ESI): $m / z[\mathrm{M}+\mathrm{H}]^{+}$ Calcd for $\mathrm{C}_{20} \mathrm{H}_{20} \mathrm{~N}_{3} \mathrm{O}_{3} \mathrm{~S}$ : 382.1225; Found: 382.1229 .

3-[3-(4-tert-Butylphenyl)-4-oxothiazolidin-2-yl]-4-phenyl-1,2,5-oxadiazole 2-oxide (7d). White solid (81\%), m.p. 150-152 ${ }^{\circ} \mathrm{C}$, IR (film): 3000, 2964, 2870, 1702, 1594, 772, $625 \mathrm{~cm}^{-1} .{ }^{1} \mathrm{H}$ NMR $\left(300 \mathrm{MHz}, \mathrm{CDCl}_{3}\right) \delta 7.56(\mathrm{tt}, 1 \mathrm{H}, J 7.4,1.8 \mathrm{~Hz}), 7.50-7.45(\mathrm{~m}, 2 \mathrm{H}), 7.38-7.36(\mathrm{~m}, 2 \mathrm{H}), 7.21$ (brd, 2H, J 7.1 Hz), 7.06-7.03 (m, 2H), $6.11(\mathrm{~s}, 1 \mathrm{H}), 4.01$ (brs, 1H), 3.75 (d, 1H, J 15.1 Hz), 1.28 (s, 9H). ${ }^{13} \mathrm{C} \mathrm{NMR}\left(75 \mathrm{MHz}, \mathrm{CDCl}_{3}\right) \delta 170.5,155.8,151.7,133.0,131.2,129.1,127.9,126.6$, 125.1, 125.0, 114.4, 64.0, 49.9, 30.9, 24.8. ESI-MS: $m / z 396(\mathrm{M}+\mathrm{Na})^{+}$. HRMS (ESI): $m / z[\mathrm{M}+$ $\mathrm{H}]^{+}$Calcd for $\mathrm{C}_{21} \mathrm{H}_{22} \mathrm{~N}_{3} \mathrm{O}_{3} \mathrm{~S}$ : 396.1379; Found: 396.1376.

3-[3-(3,5-Dimethylphenyl)-4-oxothiazolidin-2-yl]-4-phenyl-1,2,5-oxadiazole 2-oxide (7e). White solid (78\%), m.p. 160-161 ${ }^{\circ} \mathrm{C}$, IR (film): 2921, 2852, 1681, 1598, 1384, 1217, 1024, 860, $768 \mathrm{~cm}^{-1} .{ }^{1} \mathrm{H}$ NMR $\left(300 \mathrm{MHz}, \mathrm{CDCl}_{3}\right) \delta 7.57$ (tt, $1 \mathrm{H}, J$ 7.6, $\left.1.8 \mathrm{~Hz}\right), 7.49(\mathrm{~m}, 2 \mathrm{H}), 7.27$ (brd, 2H, $J 7.2 \mathrm{~Hz}), 6.92(\mathrm{~s}, 1 \mathrm{H}), 6.70(\mathrm{~s}, 2 \mathrm{H}), 6.13(\mathrm{~s}, 1 \mathrm{H}), 4.05$ (brd, 1H, J $14.6 \mathrm{~Hz}), 3.75$ (d, 1H, J 14.6 $\mathrm{Hz}), 2.23(\mathrm{~s}, 6 \mathrm{H}) .{ }^{13} \mathrm{C} \mathrm{NMR}\left(75 \mathrm{MHz}, \mathrm{CDCl}_{3}\right) \delta 170.1,155.9,139.6,135.8,131.3,130.3,129.3$, 128.1, 125.4, 123.1, 114.5, 54.8, 33.9, 21.2. ESI-MS: $m / z 390(\mathrm{M}+\mathrm{Na})^{+}$. HRMS (ESI): $m / z$ [M + $\mathrm{H}]^{+}$Calcd for $\mathrm{C}_{19} \mathrm{H}_{18} \mathrm{~N}_{3} \mathrm{O}_{3} \mathrm{~S}: 368.1063$; Found: 368.1056 .

3-[3-(3,4-Dimethylphenyl)-4-oxothiazolidin-2-yl]-4-phenyl-1,2,5-oxadiazole 2-oxide (7f). White solid (78\%), m.p. $145-147{ }^{\circ} \mathrm{C}$, IR (film): 3421, 2922, 1594, 1136, 785, $631 \mathrm{~cm}^{-1} .{ }^{1} \mathrm{H}$ NMR $\left(300 \mathrm{MHz}, \mathrm{CDCl}_{3}\right) \delta 7.59-7.55(\mathrm{~m}, 1 \mathrm{H}), 7.49(\mathrm{t}, 2 \mathrm{H}, J 7.4 \mathrm{~Hz}), 7.28(\mathrm{~d}, 2 \mathrm{H}, J 7.0 \mathrm{~Hz}), 7.11(\mathrm{~d}, 1 \mathrm{H}$, $J 8.2 \mathrm{~Hz}), 6.87-6.84(\mathrm{~m}, 2 \mathrm{H}), 6.13(\mathrm{~s}, 1 \mathrm{H}), 3.75(\mathrm{~d}, 1 \mathrm{H}, J 15.2 \mathrm{~Hz}), 2.22(\mathrm{~s}, 3 \mathrm{H}), 2.18(\mathrm{~s}, 3 \mathrm{H}), 4.02$ (brs, $1 \mathrm{H}) .{ }^{13} \mathrm{C}$ NMR $\left(75 \mathrm{MHz}, \mathrm{CDCl}_{3}\right) \delta 170.1,155.8,138.3,137.3,133.5,131.2,130.7,129.2$, 128.0, 126.5, 125.3, 122.6, 114.4, 54.7, 33.8, 19.7, 19.4. ESI-MS: $m / z 390(\mathrm{M}+\mathrm{Na})^{+}$. HRMS (ESI): $m / z[\mathrm{M}+\mathrm{Na}]^{+}$Calcd for $\mathrm{C}_{19} \mathrm{H}_{17} \mathrm{~N}_{3} \mathrm{O}_{3} \mathrm{SNa}$ : 390.0890; Found: 390.0889 .

3-[3-(4-Methoxyphenyl)-4-oxothiazolidin-2-yl]-4-phenyl-1,2,5-oxadiazole 2-oxide (7g). Brown solid (78\%), m.p. 129-131 ${ }^{\circ} \mathrm{C}$, IR (film): 2922, 2851, 1693, 1594, 1235, 1017, 840, 766, $699 \mathrm{~cm}^{-1} .{ }^{1} \mathrm{H}$ NMR $\left(300 \mathrm{MHz}, \mathrm{CDCl}_{3}\right) \delta$ 7.61-7.54 (m, 1H), 7.53-7.46 (m, 2H), 7.30-7.24 (m, 2H), 7.05 (d, 2H, J 8.7 Hz), 6.87 (d, 2H, J 8.7 Hz), 6.08 (s, 1H), 4.05 (brd, 1H, J 13.9 Hz), 3.76 (d, 1H, $J 13.9 \mathrm{~Hz}), 3.78(\mathrm{~s}, 3 \mathrm{H}) .{ }^{13} \mathrm{C} \mathrm{NMR}\left(75 \mathrm{MHz}, \mathrm{CDCl}_{3}\right) \delta 170.3,159.3,155.7,131.2,129.2,128.4$, 
127.9, 127.0, 125.1, 114.9, 114.3, 55.3, 54.8, 33.6. ESI-MS: $\mathrm{m} / z 370(\mathrm{M}+\mathrm{H})^{+}$. HRMS (ESI): $\mathrm{m} / z$ $[\mathrm{M}+\mathrm{H}]^{+}$Calcd for $\mathrm{C}_{18} \mathrm{H}_{16} \mathrm{~N}_{3} \mathrm{O}_{4} \mathrm{~S}$ : 370.0856 ; Found: 370.0860 .

3-[3-(3,4-Dimethoxyphenyl)-4-oxothiazolidin-2-yl]-4-phenyl-1,2,5-oxadiazole 2-oxide (7h). White solid (77\%), m.p. 120-124 ${ }^{\circ} \mathrm{C}$, IR (film): 2926, 1692, 1585, 1216, 1017, 936, 768, $682 \mathrm{~cm}^{-1}$. ${ }^{1} \mathrm{H}$ NMR $\left(300 \mathrm{MHz}, \mathrm{CDCl}_{3}\right) \delta$ 7.60-7.45 (m, 3H), $7.28(\mathrm{~d}, 2 \mathrm{H}, J 7.5 \mathrm{~Hz}), 6.81(\mathrm{~d}, 1 \mathrm{H}, J 8.4 \mathrm{~Hz})$, 6.68 (dd, 1H, J 8.4, $2.2 \mathrm{~Hz}), 6.61$ (d, 1H, J 2.2 Hz), 6.11 (s, 1H), 4.06 (brd, 1H, J $14.9 \mathrm{~Hz}), 3.86$ $(\mathrm{s}, 3 \mathrm{H}), 3.77$ (d, $1 \mathrm{H}, J 14.9), 3.74$ (s, 3H). ${ }^{13} \mathrm{C} \mathrm{NMR}\left(75 \mathrm{MHz}, \mathrm{CDCl}_{3}\right) \delta 170.2,155.7,149.6,149.0$, 131.3, 129.3, 128.7, 128.0, 125.3, 118.0, 114.4, 111.4, 108.9, 55.9, 55.9, 54.8, 33.7. ESI-MS: $\mathrm{m} / \mathrm{z}$ $400(\mathrm{M}+\mathrm{H})^{+}$. HRMS (ESI): $m / z[\mathrm{M}+\mathrm{H}]^{+}$Calcd for $\mathrm{C}_{19} \mathrm{H}_{18} \mathrm{~N}_{3} \mathrm{O}_{5} \mathrm{~S}: 400.0958$; Found: 400.0955 .

3-[3-(1,3-Benzodioxol-5-yl)-4-oxothiazolidin-2-yl]-4-phenyl-1,2,5-oxadiazole 2-oxide (7i). White solid (77\%), m.p. 121-123 ${ }^{\circ} \mathrm{C}$, IR (film): 3003, 2965, 2930, 1697, 1591, 833, $779 \mathrm{~cm}^{-1} \cdot{ }^{1} \mathrm{H}$ NMR (300 MHz, $\left.\mathrm{CDCl}_{3}\right) \delta 7.58(\mathrm{tt}, 1 \mathrm{H}, J 1.9,7.4 \mathrm{~Hz}), 7.53-7.49$ (m, 2H), 7.32 (brd, 2H, $J 7.3$ Hz), 6.76 (d. 1H, J 8.2 Hz), 6.63 (d, 1H, J 1.9 Hz), 6.57 (dd, 1H, J 8.2, 1.9 Hz), 6.05 (s, 1H), 5.99$5.98(\mathrm{~m}, 2 \mathrm{H}), 4.04$ (brd, 1H, $J 15.2 \mathrm{~Hz}), 3.75$ (d, $1 \mathrm{H}, J 15.2 \mathrm{~Hz}) .{ }^{13} \mathrm{C} \mathrm{NMR}\left(75 \mathrm{MHz}, \mathrm{CDCl}_{3}\right) \delta$ 170.4, 155.6, 148.5, 147.7, 131.3, 129.5, 129.3, 127.9, 125.1, 119.4, 114.3, 108.6, 107.1, 101.8, 64.2, 33.6. ESI-MS: $m / z 384(\mathrm{M}+\mathrm{H})^{+}$. HRMS (ESI): $m / z[\mathrm{M}+\mathrm{H}]^{+}$Calcd for $\mathrm{C}_{18} \mathrm{H}_{14} \mathrm{~N}_{3} \mathrm{O}_{5} \mathrm{~S}$ : 384.0648; Found: 384.0658 .

3-[3-(3-Fluorophenyl)-4-oxothiazolidin-2-yl]-4-phenyl-1,2,5-oxadiazole 2-oxide (7j). Brown solid (77\%), m.p. 99-101 ${ }^{\circ} \mathrm{C}$, IR (film): 2924, 1595, 1376, $1020 \mathrm{~cm}^{-1} .{ }^{1} \mathrm{H}$ NMR (300 MHz, $\left.\mathrm{CDCl}_{3}\right)$ $\delta 7.60$ (tt, $1 \mathrm{H}, J 7.3,1.3 \mathrm{~Hz}), 7.56-7.51$ (m, 2H), 7.33 (brd, 2H, J 8.2 Hz), 7.32-7.29 (m, 1H), 7.00 (dt, 1H, J 7.4, $1.8 \mathrm{~Hz}), 6.94-6.89$ (m, 2H). 6.19 (s, 1H), 3.99 (brd, 1H, $J 15.4 \mathrm{~Hz}), 3.77$ (d, 1H, $J$ $15.4 \mathrm{~Hz}) .{ }^{13} \mathrm{C}$ NMR $\left(75 \mathrm{MHz}, \mathrm{CDCl}_{3}\right) \delta 169.9,162.8$ (d, $\left.J 249.2 \mathrm{~Hz}\right), 155.6,137.5$ (d, $\left.J=9.9 \mathrm{~Hz}\right)$, 131.4, 130.8 (d, $J=8.8 \mathrm{~Hz}), 129.4,128.0,125.1,120.1,115.3$ (d, $J 21.5 \mathrm{~Hz}), 114.4,112.6$ (d, $J$ $24.2 \mathrm{~Hz})$, 54.4, 33.7. ESI-MS: $m / z 358(\mathrm{M}+\mathrm{H})^{+}$. HRMS (ESI): $m / z[\mathrm{M}+\mathrm{H}]^{+}$Calcd for $\mathrm{C}_{17} \mathrm{H}_{13} \mathrm{FN}_{3} \mathrm{O}_{3} \mathrm{~S}$ : 358.0656 ; Found: 358.0661 .

3-[3-(4-Fluorophenyl)-4-oxothiazolidin-2-yl]-4-phenyl-1,2,5-oxadiazole 2-oxide (7k). White solid (77\%), m.p. 118-120 ${ }^{\circ} \mathrm{C}$, IR (film): 3019, 2927, 1697, 1598, 1509, 1383, 1217, 825, $767 \mathrm{~cm}^{-}$ ${ }^{1} .{ }^{1} \mathrm{H}$ NMR $\left(300 \mathrm{MHz}, \mathrm{CDCl}_{3}\right) \delta$ 7.62-7.48 (m, 3H), 7.29 (brd, 2H, J 7.5 Hz), 7.14-7.00 (m, 4H), $6.13(\mathrm{~s}, 1 \mathrm{H}), 4.04$ (brd, $1 \mathrm{H}, J 15.1 \mathrm{~Hz}), 3.77$ (d, $1 \mathrm{H}, J 15.1 \mathrm{~Hz}) .{ }^{13} \mathrm{C} \mathrm{NMR}\left(75 \mathrm{MHz}, \mathrm{CDCl}_{3}\right) \delta$ 170.2, 161.8 (d, $J 248.8 \mathrm{~Hz}$ ), 155.6, 131.9 (d, $J 2.7 \mathrm{~Hz}), 131.4,129.4,127.9,127.5$ (d, J 9.1 Hz), 125.1, 116.9 (d, $J 24.7 \mathrm{~Hz}), 114.2,54.6,33.6$. ESI-MS: $m / z 358(\mathrm{M}+\mathrm{H})^{+}$. HRMS (ESI): $m / z$ [M + $\mathrm{H}]^{+}$Calcd for $\mathrm{C}_{17} \mathrm{H}_{13} \mathrm{FN}_{3} \mathrm{O}_{3} \mathrm{~S}$ : 358.0656; Found: 358.0660 .

3-[3-(3-Chlorophenyl)-4-oxothiazolidin-2-yl]-4-phenyl-1,2,5-oxadiazole 2-oxide (71). White solid (80\%), m.p. 108-110 ${ }^{\circ} \mathrm{C}$, IR (film): 2922, 2852, 1697, 1593, 1387, 1019, 769, 723, $692 \mathrm{~cm}^{-1}$. ${ }^{1} \mathrm{H}$ NMR (300 MHz, $\left.\mathrm{CDCl}_{3}\right) \delta$ 7.63-7.50 (m, 3H), 7.32 (brd, 2H, J 6.9 Hz), 7.29-7.25 (m, 2H), $7.11(\mathrm{~s}, 1 \mathrm{H}), 7.06-7.01(\mathrm{~m}, 1 \mathrm{H}), 6.18(\mathrm{~s}, 1 \mathrm{H}), 4.02(\mathrm{brd}, 1 \mathrm{H}, J 15.4 \mathrm{~Hz}), 3.76(\mathrm{~d}, 1 \mathrm{H}, J 15.4 \mathrm{~Hz})$. ${ }^{13} \mathrm{C} \mathrm{NMR}\left(75 \mathrm{MHz}, \mathrm{CDCl}_{3}\right) \delta 169.9,155.6,137.1,135.1,131.4,130.5,129.4,128.3,128.0,125.3$, 125.1, 122.8, 114.2, 54.3, 33.6. ESI-MS: $m / z$ 374, (M+H) ${ }^{+}$. HRMS (ESI): $m / z[\mathrm{M}+\mathrm{H}]^{+} \mathrm{Calcd}$ for $\mathrm{C}_{17} \mathrm{H}_{13}{ }^{35} \mathrm{ClN}_{3} \mathrm{O}_{3} \mathrm{~S}$ : 374.0360; Found: 374.0369; Calcd for $\mathrm{C}_{17} \mathrm{H}_{13}{ }^{37} \mathrm{ClN}_{3} \mathrm{O}_{3} \mathrm{~S}$ : 376.0332; Found: 376.0336 . 
3-[3-(4-Chlorophenyl)-4-oxothiazolidin-2-yl]-4-phenyl-1,2,5-oxadiazole 2-oxide (7m). White solid (81\%), m.p. $158-159{ }^{\circ} \mathrm{C}$, IR (film): 2924, 2853, 1697, 1575, 1387, 761, $722 \mathrm{~cm}^{-1} .{ }^{1} \mathrm{H}$ NMR $\left(300 \mathrm{MHz}, \mathrm{CDCl}_{3}\right) \delta 7.59$ (tt, 1H, J 7.4, $\left.1.3 \mathrm{~Hz}\right), 7.55-7.51(\mathrm{~m}, 2 \mathrm{H}), 7.34-7.30(\mathrm{~m}, 4 \mathrm{H}), 7.07$ (d, $2 \mathrm{H}, J 8.6 \mathrm{~Hz}), 6.17$ (s, 1H), 4.00 (brd, $1 \mathrm{H}, J 15.2), 3.76$ (d, $1 \mathrm{H}, J 15.2 \mathrm{~Hz}) .{ }^{13} \mathrm{C} \mathrm{NMR}(75 \mathrm{MHz}$, $\left.\mathrm{CDCl}_{3}\right) \delta 170.0,155.7,137.1,131.6,130.7,129.5,128.5,128.1,125.4,125.1,123.0,114.3,54.4$, 33.8. ESI-MS: $m / z$ 374, $(\mathrm{M}+\mathrm{H})^{+}$. HRMS (ESI): $m / z[\mathrm{M}+\mathrm{H}]^{+}$Calcd for $\mathrm{C}_{17} \mathrm{H}_{13}{ }^{35} \mathrm{ClN}_{3} \mathrm{O}_{3} \mathrm{~S}$ : 374.0360; Found: 374.0370; Calcd for $\mathrm{C}_{17} \mathrm{H}_{13}{ }^{37} \mathrm{ClN}_{3} \mathrm{O}_{3} \mathrm{~S}$ : 376.0332; Found: 376.0335 .

3-[3-(4-Bromophenyl)-4-oxothiazolidin-2-yl]-4-phenyl-1,2,5-oxadiazole $\quad$ 2-oxide (7n). Yellowish white solid (70\%), m.p. 198-200 ${ }^{\circ} \mathrm{C}$, IR (film): 3515, 3047, 2349, 1694, 1510, 1258, $1031 \mathrm{~cm}^{-1} .{ }^{1} \mathrm{H}$ NMR $\left(300 \mathrm{MHz}, \mathrm{CDCl}_{3}\right) \delta$ 7.63-7.58 (m, $\left.1 \mathrm{H}\right), 7.55-7.51(\mathrm{~m}, 2 \mathrm{H}), 7.47(\mathrm{~d}, 2 \mathrm{H}, J 8.6$ Hz), 7.35-7.32 (m, 2H), 7.01 (d, 2H, J 8.6 Hz), 6.18 ( s, 1H), 4.00 (brd, 1H, J $15.2 \mathrm{~Hz}$ ), 3.76 (d, $1 \mathrm{H}, J 15.2 \mathrm{~Hz}) .{ }^{13} \mathrm{C} \mathrm{NMR}\left(75 \mathrm{MHz}, \mathrm{CDCl}_{3}\right) \delta 170.0,155.6,135.0,132.8,131.5,129.4,128.0$, 126.6, 125.1, 122.0, 114.3, 54.3, 33.7. ESI-MS: $m / z 419(\mathrm{M}+\mathrm{H})^{+}$. HRMS (ESI): $m / z[\mathrm{M}+\mathrm{H}]^{+}$ Calcd for $\mathrm{C}_{17} \mathrm{H}_{13}{ }^{79} \mathrm{BrN}_{3} \mathrm{O}_{3} \mathrm{~S}$ : 417.9856; Found: 417.9869; Calcd for $\mathrm{C}_{17} \mathrm{H}_{13}{ }^{81} \mathrm{BrN}_{3} \mathrm{O}_{3} \mathrm{~S}$ : 419.9841; Found: 419.9842.

4-(4-Methoxyphenyl)-3-(4-oxo-3-phenylthiazolidin-2-yl)-1,2,5-oxadiazole 2-oxide (8a). White solid (80\%), m.p. 150-152 ${ }^{\circ} \mathrm{C}$, IR (film): 3010, 2934, 2837, 1696, 1597, 1446, 1022, 838, $745 \mathrm{~cm}^{-}$ ${ }^{1} .{ }^{1} \mathrm{H}$ NMR (300 MHz, $\left.\mathrm{CDCl}_{3}\right) \delta$ 7.60-7.46 (m, 3H), 7.30-7.24 (m, 2H), 7.05 (d, 2H, J 8.7 Hz), 6.87 (d, 2H, $J 8.7 \mathrm{~Hz}), 6.08$ (s, 1H), 4.05 (brd, 1H, J 13.8 Hz), 3.78 (s, 3H), 3.76 (d, 1H, J 13.8 $\mathrm{Hz}) .{ }^{13} \mathrm{C}$ NMR $\left(75 \mathrm{MHz}, \mathrm{CDCl}_{3}\right) \delta 170.1,161.9,155.5,136.1,129.7,129.5,128.3,125.3,117.3$, 114.7, 114.4, 55.4, 54.7, 33.8. ESI-MS: $m / z 370(\mathrm{M}+\mathrm{H})^{+}$. HRMS (ESI): $m / z[\mathrm{M}+\mathrm{H}]^{+}$Calcd for $\mathrm{C}_{18} \mathrm{H}_{16} \mathrm{~N}_{3} \mathrm{O}_{4} \mathrm{~S}: 370.0856$; Found: 370.0858.

3-[3-(4-butylphenyl)-4-oxothiazolidin-2-yl]-4-(4-methoxyphenyl)-1,2,5-oxadiazole 2-oxide (8b). White solid (81\%), m.p. 99-100 ${ }^{\circ} \mathrm{C}$, IR (film): 2958, 2933, 2856, 1691, 1596, 1448, 1252, 838, $781 \mathrm{~cm}^{-1} .{ }^{1} \mathrm{H}$ NMR $\left(300 \mathrm{MHz}, \mathrm{CDCl}_{3}\right) \delta$ 7.22-7.13 (m, 4H), $7.02(\mathrm{~d}, 2 \mathrm{H}, J 8.3 \mathrm{~Hz}), 5.97$ (d, $2 \mathrm{H}, J 8.3 \mathrm{~Hz}), 6.12$ (s, 1H), 4.06 (brd, 1H, J 15.1 Hz), 3.86 (s, 3H), 3.75 (d, 1H, J 15.1 Hz), 2.57 $(\mathrm{t}, 2 \mathrm{H}, J 7.5 \mathrm{~Hz}), 1.66-1.50(\mathrm{~m}, 2 \mathrm{H}), 1.38-1.25(\mathrm{~m}, 2 \mathrm{H}), 0.90$ (t, 3H, J 6.9 Hz). ${ }^{13} \mathrm{C}$ NMR $(75 \mathrm{MHz}$, $\left.\mathrm{CDCl}_{3}\right) \delta 170.2,161.8,155.5,143.5,133.5,129.7,129.5,125.4,117.4,114.7,114.4,55.4,54.8$, 35.1, 33.8, 33.2, 22.2, 13.8. ESI-MS: $m / z 448(\mathrm{M}+\mathrm{Na})^{+}$. HRMS (ESI): $m / z[\mathrm{M}+\mathrm{H}]^{+}$Calcd for $\mathrm{C}_{22} \mathrm{H}_{24} \mathrm{~N}_{3} \mathrm{O}_{4} \mathrm{~S}: 426.1482$; Found: 426.1480 .

3-[3-(4-Isopropylphenyl)-4-oxothiazolidin-2-yl]-4-(4-methoxyphenyl)-1,2,5-oxadiazole $\quad$ 2oxide (8c). White solid (77\%), m.p. 125-127 ${ }^{\circ} \mathrm{C}$, IR (film): 3318, 2684, 1539, 1285, 1057, $757 \mathrm{~cm}^{-}$ ${ }^{1} .{ }^{1} \mathrm{H}$ NMR (300 MHz, $\left.\mathrm{CDCl}_{3}\right) \delta$ 7.29-7.14 (m, 4H), 7.04 (d, 2H, J 8.3 Hz), 6.97 (d, 2H, J 8.3 Hz), 6.12 (s, 1H), 4.05 ( brd, 1H, J15.1 Hz), 3.86 (s, 3H), 3.76 (d, 1H, J 15.1 Hz), 2.88 (sept, 1H, J 7.5 $\mathrm{Hz}), 1.21$ (d, 6H, J 7.5 Hz). ${ }^{13} \mathrm{C}$ NMR (75 MHz, $\left.\mathrm{CDCl}_{3}\right) \delta 170.2,161.8,155.6,149.4,133.6,129.5$, $127.8,125.4,117.3,114.7,114.5,55.4,54.8,33.8,33.7,23.7$. ESI-MS: $m / z 412(\mathrm{M}+\mathrm{H})^{+}$. HRMS (ESI): $m / z[\mathrm{M}+\mathrm{H}]^{+}$Calcd for $\mathrm{C}_{21} \mathrm{H}_{22} \mathrm{~N}_{3} \mathrm{O}_{4} \mathrm{~S}: 412.1312$; Found: 412.1316.

3-[3-(4-tert-Butylphenyl)-4-oxothiazolidin-2-yl]-4-(4-methoxyphenyl)-1,2,5-oxadiazole 2oxide (8d). White solid (81\%), m.p. 126-128 ${ }^{\circ} \mathrm{C}$, IR (film): 3369, 2930, 1697, 1591, 1252, 1091, $833 \mathrm{~cm}^{-1} .{ }^{1} \mathrm{H}$ NMR $\left(300 \mathrm{MHz}, \mathrm{CDCl}_{3}\right) \delta 7.37$ (d, 2H, $\left.J 8.5 \mathrm{~Hz}\right), 7.17$ (brs, 2H), 7.05 (d, 2H, J 8.5 
Hz), 6.97 (d, 2H, J 8.6 Hz), 6.12 (s, 1H), 4.04 (brs, 1H), 3.89 (s, 3H), 3.75 (d, 1H, J 15.2 Hz), 1.28 (s, 9H). ${ }^{13} \mathrm{C}$ NMR $\left(75 \mathrm{MHz}, \mathrm{CDCl}_{3}\right) \delta 170.2,161.8,155.6,151.6,133.3,129.5,126.7,114.7$, 117.4, 114.5, 125.0, 55.4, 54.8, 34.6, 33.8, 31.1. ESI-MS: $m / z 426[\mathrm{M}+\mathrm{H}]^{+}$. HRMS (ESI): $m / z$ [M $+\mathrm{H}]^{+}$Calcd for $\mathrm{C}_{22} \mathrm{H}_{24} \mathrm{~N}_{3} \mathrm{O}_{4} \mathrm{~S}: 426.1482$; Found: 426.1484 .

3-[3-(3,5-Dimethylphenyl)-4-oxothiazolidin-2-yl]-4-(4-methoxyphenyl)-1,2,5-oxadiazole 2oxide (8e). White solid (84\%), m.p. 128-130 ${ }^{\circ} \mathrm{C}$, IR (film): 3012, 2923, 2853, 1697, 1597, 1440, 1381, 1257, 1030, 836, $756 \mathrm{~cm}^{-1} .{ }^{1} \mathrm{H}$ NMR $\left(300 \mathrm{MHz}, \mathrm{CDCl}_{3}\right) \delta 7.22$ (d, 2H, J $\left.5.9 \mathrm{~Hz}\right), 6.99$ (dt, $2 \mathrm{H}, J 8.5,2.1 \mathrm{~Hz}), 6.92$ (brs, $1 \mathrm{H}), 6.71(\mathrm{~s}, 2 \mathrm{H}), 6.13(\mathrm{~s}, 1 \mathrm{H}), 4.07$ (brs, $1 \mathrm{H}), 3.87$ (s, 3H), 3.77 (d, $1 \mathrm{H}, J 15.1 \mathrm{~Hz}), 2.24(\mathrm{~s}, 6 \mathrm{H}) .{ }^{13} \mathrm{C} \mathrm{NMR}\left(75 \mathrm{MHz}, \mathrm{CDCl}_{3}\right) \delta 170.1,161.8,155.6,139.5,135.8$, 130.2, 129.6, 123.0, 117.5, 114.7, 114.4, 55.4, 54.8, 33.9, 21.1. ESI-MS: $m / z 398(\mathrm{M}+\mathrm{H})^{+}$. HRMS (ESI): $m / z[\mathrm{M}+\mathrm{H}]^{+}$Calcd for $\mathrm{C}_{20} \mathrm{H}_{20} \mathrm{~N}_{3} \mathrm{O}_{4} \mathrm{~S}: 398.1169$; Found: 398.1169 .

3-[3-(3,4-Dimethylphenyl)-4-oxothiazolidin-2-yl]-4-(4-methoxyphenyl)-1,2,5-oxadiazole 2oxide (8f). White solid (78\%), m.p. 120-122 ${ }^{\circ} \mathrm{C}$, IR (film): 3012, 2056, 1697, 1597, 1137, 836, $593 \mathrm{~cm}^{-1} .{ }^{1} \mathrm{H}$ NMR $\left(300 \mathrm{MHz}, \mathrm{CDCl}_{3}\right) \delta 7.24(\mathrm{~d}, 2 \mathrm{H}, J 7.7 \mathrm{~Hz}), 7.10(\mathrm{~d}, 1 \mathrm{H}, J 7.7 \mathrm{~Hz}), 7.02-6.96$ (m, 2H,), $6.85(\mathrm{~m}, 2 \mathrm{H}), 6.13(\mathrm{~s}, 1 \mathrm{H}), 4.05$ (brd, 1H, J $15.1 \mathrm{~Hz}), 3.87$ (s, 3H), 3.76 (d, 1H, J 15.1 $\mathrm{Hz}), 2.21(\mathrm{~s}, 3 \mathrm{H}), 2.18(\mathrm{~s}, 3 \mathrm{H}) .{ }^{13} \mathrm{C} \mathrm{NMR}\left(75 \mathrm{MHz}, \mathrm{CDCl}_{3}\right) \delta 170.2,161.8,155.6,138.3,137.3$, 133.6, 130.7, 129.6, 126.5, 122.6, 117.5, 114.7, 114.5, 55.4, 54.8, 33.8, 19.7, 19.4. ESI-MS: $m / z$ $398(\mathrm{M}+\mathrm{H})^{+}$. HRMS (ESI): $m / z$ [M+ H] $]^{+}$Calcd for $\mathrm{C}_{20} \mathrm{H}_{20} \mathrm{~N}_{3} \mathrm{O}_{4} \mathrm{~S}: 398.1165$; Found: 398.1162 .

4-(4-Methoxyphenyl)-3-[3-(4-methoxyphenyl)-4-oxothiazolidin-2-yl]-1,2,5-oxadiazole 2oxide (8g). White solid (78\%), m.p. 112-114 ${ }^{\circ} \mathrm{C}$, IR (film): 3009, 2936, 2840, 1695, 1590, 1448, $1025,839,745 \mathrm{~cm}^{-1} .{ }^{1} \mathrm{H}$ NMR $\left(300 \mathrm{MHz}, \mathrm{CDCl}_{3}\right) \delta 7.21(\mathrm{~d}, 2 \mathrm{H}, J 8.8 \mathrm{~Hz}), 7.04(\mathrm{~d}, 2 \mathrm{H}, J 8.6 \mathrm{~Hz})$, $6.98(\mathrm{~d}, 2 \mathrm{H}, J 8.6 \mathrm{~Hz}), 6.86$ (d, 2H, J 8.8 Hz), 6.08 (s, 1H), 4.06 (brd, 1H, J 14.9 Hz), 3.86 (s, 3H), $3.77(\mathrm{~s}, 3 \mathrm{H}), 3.76(\mathrm{~d}, 1 \mathrm{H}, J 14.9 \mathrm{~Hz}) .{ }^{13} \mathrm{C} \mathrm{NMR}\left(75 \mathrm{MHz}, \mathrm{CDCl}_{3}\right) \delta 170.4,161.8,159.4,155.5$, $129.5,128.5,127.1,117.3,115.0,114.8,114.4,55.4,54.9,33.7$. ESI-MS: $m / z 400(\mathrm{M}+\mathrm{H})^{+}$. HRMS (ESI): $m / z[\mathrm{M}+\mathrm{H}]^{+}$Calcd for $\mathrm{C}_{19} \mathrm{H}_{18} \mathrm{~N}_{3} \mathrm{O}_{5} \mathrm{~S}: 400.09617$; Found: 400.0961 .

3-[3-(3,4-Dimethoxyphenyl)-4-oxothiazolidin-2-yl]-4-(4-methoxyphenyl)-1,2,5-oxadiazole 2oxide (8h). White solid (78\%), m.p. 128-130 ${ }^{\circ} \mathrm{C}$, IR (film): 3422, 2852, 1593, 1387, 1019, 692, $516 \mathrm{~cm}^{-1} .{ }^{1} \mathrm{H}$ NMR $\left(300 \mathrm{MHz}, \mathrm{CDCl}_{3}\right) \delta 7.23(\mathrm{~d}, 2 \mathrm{H}, J 8.6 \mathrm{~Hz}), 6.98(\mathrm{~d}, 2 \mathrm{H}, J 8.6 \mathrm{~Hz}), 6.80(\mathrm{~d}$, $1 \mathrm{H}, J 8.4 \mathrm{~Hz}), 6.65$ (dd, 2H, J 8.4, $2.2 \mathrm{~Hz}), 6.12(\mathrm{~s}, 1 \mathrm{H}), 4.15-3.98(\mathrm{~m}, 1 \mathrm{H}), 3.86(\mathrm{~s}, 3 \mathrm{H}), 3.85$ (s, $3 \mathrm{H}), 3.82-3.73(\mathrm{~m}, 4 \mathrm{H}) .{ }^{13} \mathrm{C} \mathrm{NMR}\left(75 \mathrm{MHz}, \mathrm{CDCl}_{3}\right) \delta 170.3,161.8,155.5,149.5,148.9,129.4$, 128.6, 118.0, 117.3, 114.7, 114.4, 111.3, 108.8, 55.9, 55.8, 55.4, 54.9, 33.7. ESI-MS: $m / z 430$ $(\mathrm{M}+\mathrm{H})^{+}$. HRMS (ESI): $m / z[\mathrm{M}+\mathrm{H}]^{+}$Calcd for $\mathrm{C}_{20} \mathrm{H}_{20} \mathrm{~N}_{3} \mathrm{O}_{6} \mathrm{~S}: 430.1059$; Found: 430.1061 .

3-[3-(1,3-Benzodioxol-5-yl)-4-oxothiazolidin-2-yl]-4-(4-methoxyphenyl)-1,2,5-oxadiazole 2oxide (8i). Brown solid (76\%), m.p. $165-168{ }^{\circ} \mathrm{C}$, IR (film): 3012, 2921, 2851, 1694, 1599, 1470, 1385, 1216, 837, $758 \mathrm{~cm}^{-1} .{ }^{1} \mathrm{H}$ NMR $\left(300 \mathrm{MHz}, \mathrm{CDCl}_{3}\right) \delta 7.25$ (brd, $\left.2 \mathrm{H}, J 8.3 \mathrm{~Hz}\right), 7.03-6.98$ (m, $2 \mathrm{H}), 6.76(\mathrm{~d}, 1 \mathrm{H}, J 8.3 \mathrm{~Hz}), 6.64(\mathrm{~d}, 1 \mathrm{H}, J 2.2 \mathrm{~Hz}), 6.58(\mathrm{dd}, 1 \mathrm{H}, J 8.3,2.2 \mathrm{~Hz}), 6.05(\mathrm{~s}, 1 \mathrm{H}), 5.98$ (s, 2H), 4.06 (brd, 1H, J 15.1 Hz), $3.87(\mathrm{~s}, 3 \mathrm{H}), 3.75$ (d, 1H, $J 15.1 \mathrm{~Hz}) .{ }^{13} \mathrm{C}$ NMR $(75 \mathrm{MHz}$, $\left.\mathrm{CDCl}_{3}\right) \delta 170.4,161.9,155.5,148.5,147.7,129.6,129.5,119.4,117.3,114.8,114.3,108.6,107.2$, 
101.9, 55.4, 55.0, 33.7. ESI-MS: $m / z 414[\mathrm{M}+\mathrm{H}]^{+}$. HRMS (ESI): $m / z[\mathrm{M}+\mathrm{H}]^{+}$Calcd for $\mathrm{C}_{19} \mathrm{H}_{16} \mathrm{~N}_{3} \mathrm{O}_{6} \mathrm{~S}: 414.0754$; Found: 414.0752 .

3-[3-(3-Fluorophenyl)-4-oxothiazolidin-2-yl]-4-(4-methoxyphenyl)-1,2,5-oxadiazole 2-oxide (8j). Brown solid (74\%), m.p. 120-122 ${ }^{\circ} \mathrm{C}$, IR (film): 2925, 2985, 1694, 1598, 1258, 1012, 760, $598 \mathrm{~cm}^{-1} .{ }^{1} \mathrm{H}$ NMR $\left(300 \mathrm{MHz}, \mathrm{CDCl}_{3}\right) \delta$ 7.33-7.27 (m, 3H), 7.03-6.97 (m, 3H), 6.95-6.89 (m, 2H), 6.20 (s, 1H), 4.03 (brd, $1 \mathrm{H}, J 15.1 \mathrm{~Hz}), 3.87$ (s, 3H), 3.77 (d, 1H, $J 15.1 \mathrm{~Hz}) .{ }^{13} \mathrm{C} \mathrm{NMR}(75 \mathrm{MHz}$, $\left.\mathrm{CDCl}_{3}\right) \delta 170.0,162.8(\mathrm{~d}, J 248.8 \mathrm{~Hz}), 161.8,155.4,137.5(\mathrm{~d}, J 9.9 \mathrm{~Hz}), 130.8(\mathrm{~d}, J 9.1 \mathrm{~Hz})$, 129.5, 120.2, 117.2, 115.3 (d, $J 21.8 \mathrm{~Hz}), 114.9,114.4,112.7$ (d, $J 23.6 \mathrm{~Hz}$ ), 55.4, 54.4, 33.7. ESIMS: $m / z 388(\mathrm{M}+\mathrm{H})^{+}$. HRMS (ESI): $m / z[\mathrm{M}+\mathrm{H}]^{+}$Calcd for $\mathrm{C}_{18} \mathrm{H}_{15} \mathrm{FN}_{3} \mathrm{O}_{4} \mathrm{~S}: 388.0761$; Found: 388.0767 .

3-[3-(4-Fluorophenyl)-4-oxothiazolidin-2-yl]-4-(4-methoxyphenyl)-1,2,5-oxadiazole 2-oxide (8k). White solid (74\%), m.p. 110-112 ${ }^{\circ} \mathrm{C}$, IR (film): 3017, 2970, 2939, 1694, 1598, 1440, 1219, 1031, 837, $768 \mathrm{~cm}^{-1} .{ }^{1} \mathrm{H}$ NMR (300 MHz, $\left.\mathrm{CDCl}_{3}\right) \delta 7.24$ (brd, 2H, $\left.J 8.6 \mathrm{~Hz}\right), 7.13-7.09(\mathrm{~m}, 2 \mathrm{H})$, 7.06-7.02 (m, 2H), 7.00 (d, 2H, J 8.6 Hz), $6.13(\mathrm{~s}, 1 \mathrm{H}), 4.06$ (brd, 1H, J 15.2 Hz), $3.87(\mathrm{~s}, 3 \mathrm{H})$, $3.77(\mathrm{~d}, 1 \mathrm{H}, J 15.2 \mathrm{~Hz}) .{ }^{13} \mathrm{C} \mathrm{NMR}\left(75 \mathrm{MHz}, \mathrm{CDCl}_{3}\right) \delta 170.4,161.9(\mathrm{~d}, J 250.2 \mathrm{~Hz}), 160.2,155.5$, 132.0 (d, J 4.4 Hz), 129.5, 127.6 (d, J 8.8 Hz), 117.2, 116.9 (d, J 21.9 Hz), 114.9, 114.3, 55.5, 54.7, 33.7. ESI-MS: $m / z 388(\mathrm{M}+\mathrm{H})^{+}$. HRMS (ESI): $m / z[\mathrm{M}+\mathrm{H}]^{+}$Calcd for $\mathrm{C}_{18} \mathrm{H}_{15} \mathrm{FN}_{3} \mathrm{O}_{4} \mathrm{~S}$ : 388.0761; Found: 388.0767.

3-[3-(3-Chlorophenyl)-4-oxothiazolidin-2-yl]-4-(4-methoxyphenyl)-1,2,5-oxadiazole 2-oxide (81). Brown solid (76\%), m.p. 125-128 ${ }^{\circ} \mathrm{C}$, IR (film): 2983, 2937, 2840, 1703, 1602, 1456, 1259, 840, $786 \mathrm{~cm}^{-1} .{ }^{1} \mathrm{H}$ NMR $\left(300 \mathrm{MHz}, \mathrm{CDCl}_{3}\right) \delta$ 7.31-7.24 (m, 4H), 7.13-7.11 (m, 1H), 7.05-7.00 (m, $3 \mathrm{H}), 6.18(\mathrm{~s}, 1 \mathrm{H}), 4.05(\mathrm{brd}, 1 \mathrm{H}, J 15.8 \mathrm{~Hz}), 3.88(\mathrm{~s}, 3 \mathrm{H}), 3.77(\mathrm{~d}, 1 \mathrm{H}, J 15.8 \mathrm{~Hz}) .{ }^{13} \mathrm{C}$ NMR $(75$ $\left.\mathrm{MHz}, \mathrm{CDCl}_{3}\right) \delta 170.1,162.0,155.5,137.2,135.2,130.6,129.6,128.4,125.5,123.0,117.1,114.9$, 114.3, 55.5, 54.4, 33.8. ESI-MS: $m / z 404[\mathrm{M}+\mathrm{H}]^{+}$. HRMS (ESI): $m / z[\mathrm{M}+\mathrm{H}]^{+}$Calcd for $\mathrm{C}_{18} \mathrm{H}_{15}{ }^{35} \mathrm{ClN}_{3} \mathrm{O}_{4} \mathrm{~S}$ : 404.0466; Found: 404.0470; Calcd for $\mathrm{C}_{18} \mathrm{H}_{15}{ }^{37} \mathrm{ClN}_{3} \mathrm{O}_{4} \mathrm{~S}$ : 406.0437; Found: 406.0434.

3-[3-(4-Chlorophenyl)-4-oxothiazolidin-2-yl]-4-(4-methoxyphenyl)-1,2,5-oxadiazole 2-oxide (8m). Brown solid (77\%), m.p. 123-125 ${ }^{\circ} \mathrm{C}$, IR (film): 3088, 2933, 2836, 1697, 1595, 1446, 1252 , 836, $788 \mathrm{~cm}^{-1} .{ }^{1} \mathrm{H}$ NMR $\left(300 \mathrm{MHz}, \mathrm{CDCl}_{3}\right) \delta$ 7.34-7.28 (m, 4H), $7.08(\mathrm{~d}, 2 \mathrm{H}, J 8.6 \mathrm{~Hz}), 7.02(\mathrm{~d}$, $2 \mathrm{H}, J 8.6 \mathrm{~Hz}), 6.18(\mathrm{~s}, 1 \mathrm{H}), 4.04$ (brd, $1 \mathrm{H}, J 15.2 \mathrm{~Hz}), 3.88(\mathrm{~s}, 3 \mathrm{H}), 3.78$ (d, $1 \mathrm{H}, J 15.2 \mathrm{~Hz}) .{ }^{13} \mathrm{C}$ NMR $\left(75 \mathrm{MHz}, \mathrm{CDCl}_{3}\right) \delta 170.1,162.0,155.4,134.6,134.0,129.8,129.5,126.4,117.2,114.9$, 114.3, 55.5, 54.5, 33.7. ESI-MS: $m / z 404[\mathrm{M}+\mathrm{H}]^{+}$. HRMS (ESI): $m / z[\mathrm{M}+\mathrm{H}]^{+}$Calcd for $\mathrm{C}_{18} \mathrm{H}_{15}{ }^{35} \mathrm{ClN}_{3} \mathrm{O}_{4} \mathrm{~S}: 404.0466$; Found: 404.0469; Calcd for $\mathrm{C}_{18} \mathrm{H}_{15}{ }^{37} \mathrm{ClN}_{3} \mathrm{O}_{4} \mathrm{~S}: 406.0437$; Found: 406.0435 .

3-[3-(4-Bromophenyl)-4-oxothiazolidin-2-yl]-4-(4-methoxyphenyl)-1,2,5-oxadiazole 2-oxide (8n). White solid (70\%), m.p. $122-124{ }^{\circ} \mathrm{C}$, IR (film): 3414, 2853, 1817, 1482, 1121, $759 \mathrm{~cm}^{-1} .{ }^{1} \mathrm{H}$ NMR (300 MHz, CDCl 3 ) $\delta 7.47$ (d, 2H, J 8.6 Hz), 7.28 (d, 2H, J 8.6 Hz), 7.04-7.00 (m, 4H), 6.18 $(\mathrm{s}, 1 \mathrm{H}), 4.03$ (brd, $1 \mathrm{H}, J 15.4 \mathrm{~Hz}), 3.88(\mathrm{~s}, 3 \mathrm{H}), 3.77$ (d, $1 \mathrm{H}, 15.4 \mathrm{~Hz}) .{ }^{13} \mathrm{C} \mathrm{NMR}\left(75 \mathrm{MHz}, \mathrm{CDCl}_{3}\right)$ $\delta 170.1,162.0,155.4,135.1,132.8,129.5,126.6,122.0,117.2,114.9,114.3,55.5,54.4,33.7$. ESI- 
MS: $m / z 448(\mathrm{M}+\mathrm{H})^{+}$. HRMS (ESI): $m / z[\mathrm{M}+\mathrm{H}]^{+}$Calcd for $\mathrm{C}_{18} \mathrm{H}_{15}{ }^{79} \mathrm{BrN}_{3} \mathrm{O}_{4} \mathrm{~S}: 447.9992$; Found: 447.9992; Calcd for $\mathrm{C}_{18} \mathrm{H}_{15}{ }^{81} \mathrm{BrN}_{3} \mathrm{O}_{4} \mathrm{~S}$ : 449.9941; Found: 449.9948.

4-[4-Fluorophenyl)-3-(4-oxo-3-phenylthiazolidin-2-yl)-1,2,5-oxadiazole 2-oxide (9a). White solid (82\%), m.p. 108-110 ${ }^{\circ} \mathrm{C}$, IR (film): 3054, 2930, 2851, 1682, 1594, 1447, 1220, 840, 750, 696 $\mathrm{cm}^{-1} .{ }^{1} \mathrm{H}$ NMR $\left(300 \mathrm{MHz}, \mathrm{CDCl}_{3}\right) \delta$ 7.39-7.35 (m, 2H), 7.33-7.29 (m, 1H), 7.28-7.24 (m, 2H), 7.21-7.17 (m, 2H), 7.13-7.10 (m, 2H), 6.14 (s, 1H), 4.04 (brd, 1H, J 15.4 Hz), 3.79 (d, 1H, J 15.4 $\mathrm{Hz}) .{ }^{13} \mathrm{C} \mathrm{NMR}\left(75 \mathrm{MHz}, \mathrm{CDCl}_{3}\right) \delta 170.1,164.5$ (d, $\left.J 252.5 \mathrm{~Hz}\right), 154.9,136.0,130.3(\mathrm{~d}, J 8.8 \mathrm{~Hz})$, 129.9, 128.6, 125.5, 121.4 (d, $J 4.4$ Hz), 116.8 (d, $J 21.9$ Hz), 114.3, 54.7, 33.8. ESI-MS: $m / z 358$ $[\mathrm{M}+\mathrm{H}]^{+}$. HRMS (ESI): $m / z[\mathrm{M}+\mathrm{H}]^{+}$Calcd for $\mathrm{C}_{17} \mathrm{H}_{13} \mathrm{FN}_{3} \mathrm{O}_{3} \mathrm{~S}: 358.0656$; Found: 358.0659 .

3-[3-(4-Butylphenyl)-4-oxothiazolidin-2-yl]-4-(4-fluorophenyl)-1,2,5-oxadiazole 2-oxide (9b). White solid (78\%), m.p. $90-93{ }^{\circ} \mathrm{C}$, IR (film): 2926, 1692, 1597, 1216, 1039, $768 \mathrm{~cm}^{-1} .{ }^{1} \mathrm{H}$ NMR $\left(300 \mathrm{MHz}, \mathrm{CDCl}_{3}\right) \delta$ 7.27-7.13 (m, 6H), $7.00(\mathrm{~d}, 2 \mathrm{H}, J 8.3 \mathrm{~Hz}), 6.09(\mathrm{~s}, 1 \mathrm{H}), 4.03(\mathrm{~d}, 1 \mathrm{H}, J 15.2$ $\mathrm{Hz}), 3.77$ (d, 1H, J 15.2 Hz), 2.57 (t, 2H, J 7.7 Hz), 1.60-1.50 (m, 2H), 1.38-1.25 (m, 2H), 0.90 (t, $3 \mathrm{H}, J 7.7 \mathrm{~Hz}) .{ }^{13} \mathrm{C} \mathrm{NMR}\left(75 \mathrm{MHz}, \mathrm{CDCl}_{3}\right) \delta 170.2,164.4(\mathrm{~d}, J 252.5 \mathrm{~Hz}), 155.0,143.8,133.5$, 130.3 (d, $J 8.8 \mathrm{~Hz}), 129.8,125.4,121.4,116.7$ (d, $J 21.9 \mathrm{~Hz}), 114.3,54.8,35.2,33.8,33.3,22.2$, 13.9. ESI-MS: $m / z 414(\mathrm{M}+\mathrm{H})^{+}$. HRMS (ESI): $m / z[\mathrm{M}+\mathrm{H}]^{+}$Calcd for $\mathrm{C}_{21} \mathrm{H}_{21} \mathrm{FN}_{3} \mathrm{O}_{3} \mathrm{~S}: 414.1282$; Found: 414.1274.

4-(4-Fluorophenyl)-3-[3-(4-isopropylphenyl)-4-oxothiazolidin-2-yl]-1,2,5-oxadiazole 2-oxide (9c). White solid (78\%), m.p. 160-161 ${ }^{\circ} \mathrm{C}$, IR (film): 3416, 2961, 1599, 1444, 1186, $842 \mathrm{~cm}^{-1} .{ }^{1} \mathrm{H}$ NMR (300 MHz, $\left.\mathrm{CDCl}_{3}\right) \delta$ 7.24-7.19 (m, 4H), 7.18-7.13 (m, 2H), $7.02(\mathrm{~d}, 2 \mathrm{H}, J 8.5 \mathrm{~Hz}), 6.09$ (s, 1H), 4.03 (brd, 1H, $J 15.1 \mathrm{~Hz}), 3.76$ (d, 1H, J 15.1 Hz), 2.88 (sept, 1H, $J 7.0 \mathrm{~Hz}), 1.21$ (d, 6H, $J$ $7.0 \mathrm{~Hz}) .{ }^{13} \mathrm{C}$ NMR $\left(75 \mathrm{MHz}, \mathrm{CDCl}_{3}\right) \delta 170.0,164.3(\mathrm{~d}, J 253.4 \mathrm{~Hz}), 154.9,149.5,133.4,130.2(\mathrm{~d}$, $J$ 9.1 Hz), 127.8, 125.3, 121.3 (d, $J 2.7 \mathrm{~Hz}), 116.5$ (d, $J 21.8 \mathrm{~Hz}), 114.2,54.7,33.6,29.5,23.7$, 23.6. ESI-MS: $m / z 400(\mathrm{M}+\mathrm{H})^{+}$. HRMS (ESI): $m / z[\mathrm{M}+\mathrm{H}]^{+}$Calcd for $\mathrm{C}_{20} \mathrm{H}_{19} \mathrm{FN}_{3} \mathrm{O}_{3} \mathrm{~S}: 400.1131$; Found: 400.1116.

3-[3-(4-(tert-Butyl)phenyl)-4-oxothiazolidin-2-yl]-4-(4-fluorophenyl)-1,2,5-oxadiazole 2-oxide (9d). White solid (76\%), m.p. 175-178 ${ }^{\circ} \mathrm{C}$, IR (film): 2992, 2965, 2934, 1696, 1589, 1384, 1222, $1020,842,782,623 \mathrm{~cm}^{-1} .{ }^{1} \mathrm{H}$ NMR $\left(300 \mathrm{MHz}, \mathrm{CDCl}_{3}\right) \delta 7.38(\mathrm{~d}, 2 \mathrm{H}, J 8.6 \mathrm{~Hz}), 7.22-7.13(\mathrm{~m}, 4 \mathrm{H})$, 7.03 (d, 2H, J 8.6 Hz), 6.08 (s, 1H), 4.02 (brd, 1H, J $15.2 \mathrm{~Hz}), 3.77$ (d, 1H, J $15.2 \mathrm{~Hz}), 1.28$ (s, 9H). ${ }^{13} \mathrm{C}$ NMR $\left(75 \mathrm{MHz}, \mathrm{CDCl}_{3}\right) \delta 170.1,164.3(\mathrm{~d}, J 253.4 \mathrm{~Hz}), 154.9,151.8,133.2,130.3(\mathrm{~d}, J$ 8.1 Hz), 126.8, 125.0, 121.4 (d, J 2.7 Hz), 116.6 (d, J21.8 Hz), 114.3, 54.7, 34.6, 33.7, 31.1. ESIMS: $m / z 414(\mathrm{M}+\mathrm{H})^{+}$. HRMS (ESI): $m / z[\mathrm{M}+\mathrm{H}]^{+}$Calcd for $\mathrm{C}_{21} \mathrm{H}_{21} \mathrm{FN}_{3} \mathrm{O}_{3} \mathrm{~S}$ : 414.1282; Found: 414.1276.

3-[3-(3,5-Dimethylphenyl)-4-oxothiazolidin-2-yl]-4-(4-fluorophenyl)-1,2,5-oxadiazole 2-oxide (9e). White solid (76\%), m.p. 178-180 ${ }^{\circ} \mathrm{C}$, IR (film): 2920, 2854, 1689, 1603, 1453, 1231, 1030, 839, 733, $622 \mathrm{~cm}^{-1} .{ }^{1} \mathrm{H}$ NMR (300 MHz, $\left.\mathrm{CDCl}_{3}\right) \delta$ 7.30-7.17 (m, 4H), ), 6.93 (brs, 1H), 6.72 (s, 2H), $6.10(\mathrm{~s}, 1 \mathrm{H}), 4.05-4.00(\mathrm{~m}, 1 \mathrm{H}), 3.74(\mathrm{~d}, 1 \mathrm{H}, J 15.2 \mathrm{~Hz}), 2.24(\mathrm{~s}, 6 \mathrm{H}) .{ }^{13} \mathrm{C} \mathrm{NMR}(75 \mathrm{MHz}$, $\left.\mathrm{CDCl}_{3}\right) \delta 169.9,164.4(\mathrm{~d}, J 253.6 \mathrm{~Hz}), 154.9,139.6,135.8,130.4(\mathrm{~d}, J 8.8 \mathrm{~Hz}), 130.2,123.0,121.6$ $(\mathrm{d}, J 2.7 \mathrm{~Hz}), 116.6(\mathrm{~d}, J 22.0 \mathrm{~Hz}), 114.2,54.7,33.8,21.1$. ESI-MS: $m / z 386(\mathrm{M}+\mathrm{H})^{+}$. HRMS (ESI): $m / z[\mathrm{M}+\mathrm{H}]^{+}$Calcd for $\mathrm{C}_{19} \mathrm{H}_{17} \mathrm{FN}_{3} \mathrm{O}_{3} \mathrm{~S}$ : 386.0969; Found: 386.0967 . 
3-[3-(3,4-Dimethylphenyl)-4-oxothiazolidin-2-yl]-4-(4-fluorophenyl)-1,2,5-oxadiazole 2-oxide (9f). White solid (79\%), m.p. $145-147{ }^{\circ} \mathrm{C}$, IR (film): 3421, 1675, 1603, 1452, 839, $585 \mathrm{~cm}^{-1} .{ }^{1} \mathrm{H}$ NMR $\left(300 \mathrm{MHz}, \mathrm{CDCl}_{3}\right) \delta$ 7.30-7.26 (m, 2H), $7.17(\mathrm{dt}, 2 \mathrm{H}, J$ 8.5, $1.8 \mathrm{~Hz}), 7.10(\mathrm{~d}, 1 \mathrm{H}, J 7.9 \mathrm{~Hz})$, 6.87-6.82 (m, 2H), $6.12(\mathrm{~s}, 1 \mathrm{H}), 4.07-3.97(\mathrm{~m}, 1 \mathrm{H}), 3.80-3.72(\mathrm{~m}, 1 \mathrm{H}), 2.21(\mathrm{~s}, 3 \mathrm{H}), 2.17(\mathrm{~s}, 3 \mathrm{H})$. ${ }^{13} \mathrm{C} \mathrm{NMR}\left(75 \mathrm{MHz}, \mathrm{CDCl}_{3}\right) \delta 169.8,164.1(\mathrm{~d}, J 253.6 \mathrm{~Hz}), 154.8,138.2,137.2,133.5,130.6$, 130.2 (d, $J 8.8 \mathrm{~Hz}), 126.3,122.5,121.4$ (d, $J 2.7 \mathrm{~Hz}), 116.5$ (d, $J 22.0 \mathrm{~Hz}), 114.2,54.6,33.6,19.5$, 19.2. ESI-MS: $m / z 386(\mathrm{M}+\mathrm{H})^{+}$. HRMS (ESI): $m / z[\mathrm{M}+\mathrm{H}]^{+}$Calcd for $\mathrm{C}_{19} \mathrm{H}_{17} \mathrm{FN}_{3} \mathrm{O}_{3} \mathrm{~S}: 386.0896$; Found: 386.0963.

4-(4-Fluorophenyl)-3-[3-(4-methoxyphenyl)-4-oxothiazolidin-2-yl]-1,2,5-oxadiazole 2-oxide (9g). White solid (77\%), m.p. 142-144 ${ }^{\circ} \mathrm{C}$, IR (film): 3005, 2934, 2842, 1683, 1592, 1440, 1228, 836, 817, $773 \mathrm{~cm}^{-1} .{ }^{1} \mathrm{H}$ NMR $\left(300 \mathrm{MHz}, \mathrm{CDCl}_{3}\right) \delta$ 7.31-7.24 (m, 2H), 7.22-7.14 (m, 2H), $7.03(\mathrm{~d}$, $2 \mathrm{H}, J 9.0 \mathrm{~Hz}), 6.87$ (d, 2H, J9.0 Hz), 6.05 (s, 1H), 4.05 (brd, 1H, J 15.2 Hz), 3.78 (s, 3H), 3.77 (d, $1 \mathrm{H}, J 15.2 \mathrm{~Hz}) .{ }^{13} \mathrm{C} \mathrm{NMR}\left(75 \mathrm{MHz}, \mathrm{CDCl}_{3}\right) \delta 170.2,164.4$ (d, $\left.J 252.5 \mathrm{~Hz}\right), 159.4,154.8,130.3$ (d, $J 8.8 \mathrm{~Hz}), 128.4,127.1,121.4,116.8$ (d, $J 21.9 \mathrm{~Hz}), 115.0,114.2,55.4,54.8,33.6$. ESI-MS: $m / z 388(\mathrm{M}+\mathrm{H})^{+}$. HRMS (ESI): $m / z[\mathrm{M}+\mathrm{H}]^{+}$Calcd for $\mathrm{C}_{18} \mathrm{H}_{15} \mathrm{FN}_{3} \mathrm{O}_{4} \mathrm{~S}:$ 388.0761; Found: 388.0758 .

3-[3-(3,4-Dimethoxyphenyl)-4-oxothiazolidin-2-yl]-4-(4-fluorophenyl)-1,2,5-oxadiazole 2-oxide (9h). White solid (77\%), m.p. 160-163 ${ }^{\circ} \mathrm{C}$, IR (film): 3353, 2966, 2044, 1684, 1445, 1099 $\mathrm{cm}^{-1} .{ }^{1} \mathrm{H}$ NMR $\left(300 \mathrm{MHz}, \mathrm{CDCl}_{3}\right) \delta$ 7.33-7.26 (m, 2H), 7.23-7.15 (m, 2H), $6.81(\mathrm{~d}, 1 \mathrm{H}, J 8.4 \mathrm{~Hz})$, 6.67 (dd, 1H, J 8.4, $2.4 \mathrm{~Hz}), 6.62$ (d, 1H, J 2.4 Hz), 6.08 (s, 1H), 4.06 (brd, 1H, J $15.4 \mathrm{~Hz}), 3.86$ $(\mathrm{s}, 3 \mathrm{H}), 3.79(\mathrm{~d}, 1 \mathrm{H}, J 15.4 \mathrm{~Hz}), 3.76(\mathrm{~s}, 3 \mathrm{H}) .{ }^{13} \mathrm{C} \mathrm{NMR}\left(75 \mathrm{MHz}, \mathrm{CDCl}_{3}\right) \delta 170.2,164.4(\mathrm{~d}, J 253.4$ Hz), 154.9, 149.6, 149.0, 130.2 (d, $J 8.2 \mathrm{~Hz}), 128.6,121.4$ (d, $J 1.8 \mathrm{~Hz}), 117.9,116.7$ (d, $J 22.7$ $\mathrm{Hz}), 114.2,111.3,108.8,55.9,55.9,54.8,33.6$. ESI-MS: $m / z 418[\mathrm{M}+\mathrm{H}]^{+}$. HRMS (ESI): $m / z$ [M $+\mathrm{H}]^{+}$Calcd for $\mathrm{C}_{19} \mathrm{H}_{17} \mathrm{FN}_{3} \mathrm{O}_{5} \mathrm{~S}: 418.0858$; Found: 418.0860 .

3-[3-(1,3-Benzodioxol-5-yl)-4-oxothiazolidin-2-yl]-4-(4-fluorophenyl)-1,2,5-oxadiazole 2-oxide (9i). White solid (74\%), m.p. 180-182 ${ }^{\circ} \mathrm{C}$, IR (film): 3069, 2908, 1681, 1587, 1444, 1038, 845, 777, 628, $669 \mathrm{~cm}^{-1} .{ }^{1} \mathrm{H}$ NMR $\left(300 \mathrm{MHz}, \mathrm{CDCl}_{3}\right) \delta$ 7.35-7.29 (m, 2H), 7.25-7.18 (m, 2H), $6.77(\mathrm{~d}$, $1 \mathrm{H}, J 8.3 \mathrm{~Hz}), 6.63(\mathrm{~d}, 1 \mathrm{H}, J 2.2 \mathrm{~Hz}), 6.57$ (dd, 1H, J 8.3, $2.2 \mathrm{~Hz}), 6.01(\mathrm{~s}, 1 \mathrm{H}), 5.99$ (s, 2H), 4.06 (brd, $1 \mathrm{H}, J 15.8 \mathrm{~Hz}), 3.77(\mathrm{~d}, 1 \mathrm{H}, J 15.8 \mathrm{~Hz}) .{ }^{13} \mathrm{C} \mathrm{NMR}\left(75 \mathrm{MHz}, \mathrm{CDCl}_{3}\right) \delta 170.4,163.8(\mathrm{~d}, J$ $251.3 \mathrm{~Hz}$ ), 148.6, 141.7, 140.8, 130.3 (d, J 8.8 Hz), 129.5, 119.5, 116.9 (d, J 21.9 Hz), 114.2, 108.8, 107.2, 102.0, 94.8, 54.9, 33.7. ESI-MS: $m / z 402[\mathrm{M}+\mathrm{H}]^{+}$. HRMS (ESI): $m / z[\mathrm{M}+\mathrm{H}]^{+} \mathrm{Calcd}$ for $\mathrm{C}_{18} \mathrm{H}_{13} \mathrm{FN}_{3} \mathrm{O}_{5} \mathrm{~S}$ : 402.0554; Found: 402.0546.

4-(4-Fluorophenyl)-3-[3-(3-fluorophenyl)-4-oxothiazolidin-2-yl]-1,2,5-oxadiazole 2-oxide (9j). White solid (73\%), m.p. 128-131 ${ }^{\circ} \mathrm{C}$, IR (film): 2928, 1680, 1387, 622, $567 \mathrm{~cm}^{-1}$. ${ }^{1} \mathrm{H}$ NMR $\left(300 \mathrm{MHz}, \mathrm{CDCl}_{3}\right) \delta$ 7.36-7.30 (m, 3H), 7.25-7.21 (m, 2H), 7.03-6.99 (m, 1H), 6.93-6.89 (m, 2H), $6.17(\mathrm{~s}, 1 \mathrm{H}), 4.01$ (brd, $1 \mathrm{H}, J 15.4 \mathrm{~Hz}), 3.80$ (d, $1 \mathrm{H}, J 15.4 \mathrm{~Hz}) \cdot{ }^{13} \mathrm{C} \mathrm{NMR}\left(75 \mathrm{MHz}, \mathrm{CDCl}_{3}\right) \delta$ 170.0, 165.0 (d, J 254.4 Hz), 162.9 (d, J 247.9 Hz), 164.5, 130.9 (d, J 8.8 Hz), 130.3 (d, J 8.8 Hz), $120.3(\mathrm{~d}, J 4.4 \mathrm{~Hz}), 117.0$ (d, $J 21.9 \mathrm{~Hz}), 116.9,115.8$ (d, J 21.9 Hz), 115.4, 114.3, 112.7 (d, $J 24.1$ Hz), 54.4, 33.8. ESI-MS: $m / z 376[\mathrm{M}+\mathrm{H}]^{+}$. HRMS (ESI): $m / z[\mathrm{M}+\mathrm{H}]^{+}$Calcd for $\mathrm{C}_{17} \mathrm{H}_{12} \mathrm{~F}_{2} \mathrm{~N}_{3} \mathrm{O}_{3} \mathrm{~S}$ : 376.0561; Found: 376.0566. 
4-(4-Fluorophenyl)-3-[3-(4-fluorophenyl)-4-oxothiazolidin-2-yl]-1,2,5-oxadiazole 2-oxide (9k). White solid (74\%), m.p. 148-150 ${ }^{\circ} \mathrm{C}$, IR (film): 3075, 2927, 2852, 1681, 1595, 1508, 1387, 1217, 843, 776, 619, $588 \mathrm{~cm}^{-1} .{ }^{1} \mathrm{H}$ NMR $\left(300 \mathrm{MHz}, \mathrm{CDCl}_{3}\right) \delta$ 7.34-7.28 (m, 2H), 7.24-7.19 (m, 2H), 7.13-7.08 (m, 2H), 7.08-7.02 (m, 2H), $6.11(\mathrm{~s}, 1 \mathrm{H}), 4.05$ (brd, 1H, J $15.2 \mathrm{~Hz}), 3.79$ (d, 1H, $J$ $15.2 \mathrm{~Hz}) .{ }^{13} \mathrm{C}$ NMR $\left(75 \mathrm{MHz}, \mathrm{CDCl}_{3}\right) \delta 170.3,164.6(\mathrm{~d}, J 254.7 \mathrm{~Hz}), 162.0(\mathrm{~d}, J 248.1 \mathrm{~Hz}), 154.8$, 131.9 (d, $J 4.4 \mathrm{~Hz}), 130.7$ (d, J 8.8 Hz), 127.5 (d, $J 6.6 \mathrm{~Hz}), 121.3,117.0$ (d, $J 21.9 \mathrm{~Hz}), 116.8$ (d, $J 21.9 \mathrm{~Hz}), 114.1,54.7,33.6$. ESI-MS: $m / z 376(\mathrm{M}+\mathrm{H})^{+}$. HRMS (ESI): $m / z[\mathrm{M}+\mathrm{H}]^{+}$Calcd for $\mathrm{C}_{17} \mathrm{H}_{12} \mathrm{~F}_{2} \mathrm{~N}_{3} \mathrm{O}_{3} \mathrm{~S}: 376.0561$; Found: 376.0564 .

3-[3-(3-Chlorophenyl)-4-oxothiazolidin-2-yl]-4-(4-fluorophenyl)-1,2,5-oxadiazole 2-oxide (9l). White solid (74\%), m.p. $105-107{ }^{\circ} \mathrm{C}$, IR (film): 3001, 2929, 1599, 1444, 850, $618 \mathrm{~cm}^{-1} .{ }^{1} \mathrm{H}$ NMR (300 MHz, $\left.\mathrm{CDCl}_{3}\right) \delta$ 7.35-7.31 (m, 2H), 7.29-7.26 (m, 2H), 7.24-7.20 (m, 2H), 7.11-7.10 (m, 1H), 7.04-7.01 (m, 1H), $6.17(\mathrm{~s}, 1 \mathrm{H}), 4.03(\mathrm{brd}, 1 \mathrm{H}, J 15.4 \mathrm{~Hz}), 3.78(\mathrm{~d}, 1 \mathrm{H}, J 15.4 \mathrm{~Hz}) .{ }^{13} \mathrm{C}$ NMR $\left(75 \mathrm{MHz}, \mathrm{CDCl}_{3}\right) \delta 170.0,166.2(\mathrm{~d}, J 252.4 \mathrm{~Hz}), 154.8,137.1,135.3,130.7,130.4(\mathrm{~d}, J 8.8$ Hz), 128.6, 125.4, 123.0, $121.2,116.9$ (d, $J 21.9 \mathrm{~Hz}), 114.2$, 54.4, 33.7. ESI-MS: $m / z 392(\mathrm{M}+\mathrm{H})^{+}$. HRMS (ESI): $m / z$ [M $+\mathrm{H}]^{+}$Calcd for $\mathrm{C}_{17} \mathrm{H}_{12} \mathrm{~F}^{35} \mathrm{ClN}_{3} \mathrm{O}_{3} \mathrm{~S}$ : 392.0266; Found: 392.0269; Calcd for $\mathrm{C}_{17} \mathrm{H}_{12} \mathrm{~F}^{37} \mathrm{ClN}_{3} \mathrm{O}_{3} \mathrm{~S}$ : 394.0237; Found: 394.0234 .

3-[3-(4-Chlorophenyl)-4-oxothiazolidin-2-yl]-4-(4-fluorophenyl)-1,2,5-oxadiazole 2-oxide (9m). White solid (82\%), m.p. 109-110 ㄷ, IR (film): 2974, 2929, 2851, 1686, 1601, 1445, 1384, 1217, 842, 786, $620 \mathrm{~cm}^{-1} .{ }^{1} \mathrm{H}$ NMR (300 MHz, $\left.\mathrm{CDCl}_{3}\right) \delta$ 7.36-7.31 (m, 4H), 7.25-7.20 (m, 2H), 7.08-7.05 (m, 2H), $6.15(\mathrm{~s}, 1 \mathrm{H}), 4.01(\mathrm{~d}, 1 \mathrm{H}, J 15.4 \mathrm{~Hz}), 3.79(\mathrm{~d}, 1 \mathrm{H}, J 15.4 \mathrm{~Hz}) .{ }^{13} \mathrm{C}$ NMR $(75$ $\left.\mathrm{MHz}, \mathrm{CDCl}_{3}\right) \delta 170.0,164.4(\mathrm{~d}, J 254.68 \mathrm{~Hz}), 154.7,134.3(\mathrm{~d}, J 21.9 \mathrm{~Hz}), 130.3(\mathrm{~d}, J 8.8 \mathrm{~Hz})$, 130.0, 129.0, 126.4, 121.3, 116.9 (d, $J 21.9 \mathrm{~Hz}$ ), 114.2, 54.4, 33.7. ESI-MS: $m / z 392[\mathrm{M}+\mathrm{H}]^{+}$. HRMS (ESI): $m / z$ [M $+\mathrm{H}]^{+}$Calcd for $\mathrm{C}_{17} \mathrm{H}_{12} \mathrm{~F}^{35} \mathrm{ClN}_{3} \mathrm{O}_{3} \mathrm{~S}$ : 392.0266; Found: 392.0269. Calcd for $\mathrm{C}_{17} \mathrm{H}_{12} \mathrm{~F}^{37} \mathrm{ClN}_{3} \mathrm{O}_{3} \mathrm{~S}$ : 394.0237; Found: 394.0235 .

3-[3-(4-Bromophenyl)-4-oxothiazolidin-2-yl]-4-(4-fluorophenyl)-1,2,5-oxadiazole 2-oxide (9n). Brown solid (70\%), m.p. 147-150 ${ }^{\circ} \mathrm{C}$, IR (film): 3356, 2929, 1686, 1443, 1287, 1162, 587 $\mathrm{cm}^{-1} .{ }^{1} \mathrm{H}$ NMR $\left(300 \mathrm{MHz}, \mathrm{CDCl}_{3}\right) \delta 7.48(\mathrm{~d}, 2 \mathrm{H}, J 8.6 \mathrm{~Hz}), 7.38-7.32(\mathrm{~m}, 2 \mathrm{H}), 7.26-7.19(\mathrm{~m}, 2 \mathrm{H})$, 7.01 (d, 2H, $J 8.6 \mathrm{~Hz}), 6.16$ (s, 1H), 4.01 (d, 1H, $J 15.4 \mathrm{~Hz}), 3.79$ (d, 1H, $J 15.4 \mathrm{~Hz}) .{ }^{13} \mathrm{C}$ NMR $(75$ $\left.\mathrm{MHz}, \mathrm{CDCl}_{3}\right) \delta 169.9,164.1(\mathrm{~d}, J 254.7 \mathrm{~Hz}), 154.8,134.9,132.9,130.3(\mathrm{~d}, J 8.7 \mathrm{~Hz}), 126.6,122.1$, 121.3, 116.9 (d, $J 21.9 \mathrm{~Hz}), 114.3$ 54.3, 33.6. ESI-MS: $m / z 459[\mathrm{M}+\mathrm{Na}]^{+}$. HRMS (ESI): $m / z$ [M + $\mathrm{Na}]^{+}$Calcd for $\mathrm{C}_{17} \mathrm{H}_{11} \mathrm{~F}^{79} \mathrm{BrN}_{3} \mathrm{O}_{3} \mathrm{SNa}$ : 457.9581; Found: 457.9585; Calcd for $\mathrm{C}_{17} \mathrm{H}_{11} \mathrm{~F}^{81} \mathrm{BrN}_{3} \mathrm{O}_{3} \mathrm{SNa}$ : 459.9561; Found: 459.9555.

\section{Acknowledgements}

The authors thank the Director, IICT for support. $12^{\text {th }} 5$ Yr Plan Project ORIGIN-CSC-0108 is acknowledged for financial assistance. SNK, CHNSSPK thank CSIR-New Delhi, SRVA thank NIPER-HYD, KKS thank DST-INSPIRE for the fellowship. 


\section{References}

1. Gasco, A.; Boulton. A. J. Adv. Heterocycl. Chem., 1981, 29, 251.

http://dx.doi.org/10.1016/S0065-2725(08)60789-8

2. Cerecetto, H.; Porcal, W. Mini Rev. Med. Chem., 2005, 5, 57.

http://dx.doi.org/10.2174/1389557053402864

3. Khmelnitskii, L. I.; Novikov, S. S.; Godovikova, T. I. Chemistry of Furoxans: Reactions and Applications $2^{\text {nd }}$ ed., M. Nauka, (in Russian) 1996.

4. Bohn, H.; Brendel, J.; Martorana, P. A.; Schönafinger, K. Br. J. Pharmacol.,1995, 114, 1605.

http://dx.doi.org/10.1111/j.1476-5381.1995.tb14946.x

5. Sayed, A. A.; Simeonov, A.; Thomas, C. J.; Inglese, J.; Austin, C. P.; Williams, D. L. Nat. Med., 2008, 14, 407.

http://dx.doi.org/10.1038/nm1737

6. Wang, P. G.; Xian, M.; Tang, X.; Wu, X.; Wen, Z.; Cai, T.; Janczuk, A. J. Chem. Rev., 2002, 102, 1091.

http://dx.doi.org/10.1021/cr0000401

7. Vo, D.; Nguyen, J. -T.; McEwen, C. -A.; Shan, R.; Knaus, E. E. Drug Dev. Res., 2002, 56, 1.

http://dx.doi.org/10.1002/ddr.10050

8. Boschi, D.; Tron, G. C.; Stilo, A. D.; Fruttero, R.; Gasco, A.; Poggesi, E.; Motta, G.;

Leonardi. A. J. Med. Chem., 2003, 46, 3762.

http://dx.doi.org/10.1021/jm030825u

9. Sorba, G.; Galli, U.; Cena, C.; Fruttero, R.; Gasco, A.; Morini, G.; Adami, M.; Coruzzi, G.; Brenciaglia, M. I.; Dubini, F. Chem. Bio. Chem., 2003, 4, 899.

http://dx.doi.org/10.1002/cbic.200300617

10 Tripathi, A. C.; Gupta, S. J.; Fatima, G. N.; Sonar, P. K.; Verma, A.; Saraf, S. K. Eur. J. Med. Chem., 2014, 72, 52.

http://dx.doi.org/10.1016/j.ejmech.2013.11.017

11. Verma, A.: Saraf, S. K. Eur. J. Med. Chem., 2008, 43, 897.

http://dx.doi.org/10.1016/j.ejmech.2007.07.017

12. Revelant, G.; Huber-Villaume, S.; Dunand, S.; Kirsch, G.; Schohn, H.; Hesse, S. Eur. J. Med. Chem., 2015, 94, 102.

http://dx.doi.org/10.1016/j.ejmech.2015.02.053

13. Nepali, K.; Sharma, S.; Sharma, M.; Bedi, P. M. S.; Dhar, K. L. Eur. J. Med. Chem., 2014, $77,422$.

http://dx.doi.org/10.1016/j.ejmech.2014.03.018

14. Ergenç, N.; Capan, G. Farmaco, 1994, 49, 133. 
15. Ulusoy, N.; Ergenç, N.; Ekinci, A. C.; Özer, H. Monatsh. Chem., 1996, 127, 1197. http://dx.doi.org/10.1007/BF00844695

16. Ragab, F. A.; Eid, N. M.; el-Tawab, H. A. Pharmazie, 1997, 52, 926.

17. Giri, S.; Shukla, A. K.; Nizamuddin. J. Indian. Pharm. Sci.,1990, 52, 108.

18. Cesur, N.; Cesur, Z.; Ergenc, N.; Uzun, M.; Kiraz, M.; Kasimoglu, O.; Kaya, D. Arch. Pharm.,1994, 327, 271. http://dx.doi.org/10.1002/ardp.19943270414

19. Karali, N.; Ilhan, E.; Gursoy, A.; Kiraz, M. Farmaco, 1998, 53, 346. http://dx.doi.org/10.1016/S0014-827X(98)00032-9

20. Babaoglou, K.; Page, M. A.; Jones, V. C.; McNeil, M. R.; Dong, C.; Naismith, J. H.; Lee, R. E. Bioorg. Med. Chem. Lett., 2003, 13, 3227. http://dx.doi.org/10.1016/S0960-894X(03)00673-5

21. Ulsoy, N. Arzneim.-Forsch./Drug. Res., 2002, 52, 565.

22. Anders, C. J.; Bronson, J. J.; Andrea, S. V.; Deshpande, M. S.; Falk, P. J.; Grant-Young, K. A.; Harte, E. W.; Ho, H. T.; Misco, P. F.; Robertson, J. G.; Stock, D.; Sun. Y.; Walsh, A. W. Bioorg. Med. Chem., 2000, 10, 715.

23. Mahran, M. A.; EI-Nassy, S. M. F.; Allam, S. R. Pharmazie, 2003, $58,527$.

24. Suzuki, M.; Morita, K.; Yukioka, H.; Miki, N.; Mizutani, A. J. Pestic. Sci., 2003, 28, 37. http://dx.doi.org/10.1584/jpestics.28.37

25. EI-Ansary, A. K.; Omar, A. H. Bull. Fac. Pharm. Cairo Univ., 2001, 39, 17; Chem. Abstr., 2001, 136, 216712h.

26. Schenone, S.; Bruno, O.; Ranise, A.; Bondavalli, F.; Filippeli, W.; Falcone, G.; Giordano, L.; Vitelli, M. R. Bioorg. Med. Chem., 2001, 9, 2149. http://dx.doi.org/10.1016/S0968-0896(01)00121-3

27. Bhatt, J.; Shah, B. R.; Shah, H. P.; Trivedi, P. B.; Undavia, N. K.; Desai, N. C. Indian J. Chem., 1994, 33B, 189.

28. Gududuru, V.; Hurth, E.; Dalton, J. T.; Miller, D. D. J. Med. Chem.,2005, 48, 2584. http://dx.doi.org/10.1021/jm049208b

29. Küçükgüzel, G.; Kocatepe, A.; Clercq, E. D.; Sahin, F.; Güllüce, M. Eur. J. Med. Chem., 2006, $41,353$.

http://dx.doi.org/10.1016/j.ejmech.2005.11.005

30. Tenorio, R. P.; Carvalho. C. S.; Pessanha, C. S.; Lima, J. G. De.; Edesiot, A. J.; Melo, J. T.; Goes, A. J. S. Bioorg. Med. Chem., 2005, 15, 2575.

31. Bonde, C. G.; Gaikwad, N. J. Bioorg. Med. Chem., 2004, 12, 2151. http://dx.doi.org/10.1016/j.bmc.2004.02.024

32. Barreca, M. L.; Chimirri, A.; Luca, L. D.; Monforte, A -M.; Monforte, P.; Rao, A.; Zappalà, M.; Balzarini, J.; Clercq, E. D.; Pannecouque, C.; Witvrouw, M. Bioorg. Med. Chem. Lett., 2001, 11, 1793.

http://dx.doi.org/10.1016/S0960-894X(01)00304-3 
33. Rawal, R. K.; Prabhakar, Y. S.; Katti, S. B.; Clercq, D. Bioorg. Med. Chem., 2005, 13, 6771.

http://dx.doi.org/10.1016/j.bmc.2005.07.063

34. Dayam, R.; Sanchez, T.; Clement, O.; Shoemaker, R.; Sei, S.; Nemati, N. J. Med. Chem., 2005, 48, 111.

http://dx.doi.org/10.1021/jm0496077

35. Kumar, C. N. S. S. P.; Parida, D. K.; Santhoshi, A.; Kota, A. K.; Sridhar, B.; Jayathirtha Rao, V. Med Chem Comm., 2011, 2, 486 and references cited therein.

http://dx.doi.org/10.1039/c0md00263a

36. Narender Reddy, T.; Ravinder, M.; Bagul, P.; Ravikanti, K.; Bagul, C.; Nanubolu, J. B.; Srinivas, K.; Banarjee, S. K.; Jayathirtha Rao, V. Eur. J. Med. Chem., 2014, 71, 53 and references cited therein.

http://dx.doi.org/10.1016/j.ejmech.2013.10.043

37. Gasco, A. M.; Fruttero, R.; Sorba, G.; Gasco, A. Liebigs Ann. Chem.,1991, 1211. http://dx.doi.org/10.1002/jlac.1991199101207

38. SMART \& SAINT. Software Reference manuals. Versions 6.28a \& 5.625, Bruker Analytical X-ray Systems Inc., Madison, Wisconsin, U.S.A., 2001.

39. Sheldrick, G. M. SHELXS97 and SHELXL97, Programs for crystal structure solution and refinement; University of Gottingen: Germany, 1997. 